\title{
COHOMOLOGY THEORY OF LIE GROUPS AND LIE ALGEBRAS
}

\author{
BY \\ CLAUDE CHEVALLEY AND SAMUEL EILENBERG
}

\section{INTRODUCTION}

The present paper lays no claim to deep originality. Its main purpose is to give a systematic treatment of the methods by which topological questions concerning compact Lie groups may be reduced to algebraic questions concerning Lie algebras $\left({ }^{1}\right)$. This reduction proceeds in three steps: (1) replacing questions on homology groups by questions on differential forms. This is accomplished by de Rham's theorems $\left({ }^{2}\right.$ ) (which, incidentally, seem to have been conjectured by Cartan for this very purpose); (2) replacing the consideration of arbitrary differential forms by that of invariant differential forms: this is accomplished by using invariant integration on the group manifold; (3) replacing the consideration of invariant differential forms by that of alternating multilinear forms on the Lie algebra of the group.

We study here the question not only of the topological nature of the whole group, but also of the manifolds on which the group operates. Chapter I is concerned essentially with step 2 of the list above (step 1 depending here, as in the case of the whole group, on de Rham's theorems). Besides considering invariant forms, we also introduce "equivariant" forms, defined in terms of a suitable linear representation of the group; Theorem 2.2 states that, when this representation does not contain the trivial representation, equivariant forms are of no use for topology; however, it states this negative result in the form of a positive property of equivariant forms which is of interest by itself, since it is the key to Levi's theorem (cf. later).

Chapter II is concerned with step 3 of the above list. It is then necessary to assume that the group operates transitively on the manifold under consideration, that is, that this manifold is a homogeneous space relative to the group. Theorem 13.1, in connection with Theorem 2.3, indicates a method by which the Betti numbers of any homogeneous space attached to a connected compact Lie group may be computed algebraically. However, applications of this theorem are still lacking. In particular, it is desirable to obtain an algebraic proof of Samelson's theorem $\left({ }^{3}\right)$ to the effect that, if a closed subgroup

Presented to the Society, December 29, 1946; received by the editors January 13, 1947.

(1) These methods are due to E. Cartan, Sur les invariants integraux de certains espaces homogènes clos, Annales Société Polonaise de Mathématique vol. 8 (1929) pp. 181-225. They were used by $R$. Brauer to determine the Betti numbers of the classical groups (C. R. Acad. Sci. Paris vol. 201 (1935) pp. 419-421).

(2) G. de Rham, Sur l'analysis situs des varietes a $n$ dimensions, J. Math. Pures Appl. vol. 10 (1931) pp. 115-200.

(3) H. Samelson, Beiträge zur Topologie der Gruppen-Mannigfaltigkeiten, Ann. of Math. vol. 42 (1941) pp. 1091-1137; Satz VI, p. 1134. 
$H$ of a connected compact Lie group $G$ is not homologous to 0 , then the cohomology ring of $G$ is the product of the cohomology rings of $H$ and $G / H$.

The topological questions on compact Lie groups, once they have been reduced to algebraic questions on Lie algebras, suggest a certain number of purely algebraic objects, which may be constructed in relation with any Lie algebra over a field of characteristic zero. One arrives in this way to the notion of the cohomology groups of an arbitrary Lie algebra $L$, which is the object of Chapters III and IV (Chapter III is concerned with the cohomology groups which correspond to invariant forms, Chapter IV with those which correspond to equivariant forms). Properties of these cohomology groups may be derived either from transcendental properties of compact groups (such properties apply only to semi-simple Lie algebras and are then obtained by making use of $\mathrm{H}$. Weyl's "unitary trick") or purely algebraically. Thus, Whitehead's algebraic lemma (4) (iwhich is used to prove Levi's theorems) states in our terminology that the second cohomology groups of any semisimple Lie algebra always reduce to $\{0\}$. The first cohomology groups also reduce to $\{0\}$, and this fact can be used to prove algebraically the full reducibility of representations of semi-simple Lie algebras (cf. the paper of Hochschild quoted above). In general, the second cohomology group of any Lie algebra $L$ (with respect to the trivial representation) is the dual space of the full exterior center of $L$, a notion which was introduced by $\operatorname{Ado}\left({ }^{5}\right)$. The theorem proved by Ado in this connection can be restated by saying that if $L \neq\{0\}$ is nilpotent, then its second cohomology group is not equal to $\{0\}$. Finally, we show (following Cartan) that the third cohomology group of a semi-simple algebra $L \neq\{0\}$ never reduces to $\{0\}$, which proves that the third Betti number of a compact connected semi-simple Lie group is always not equal to 0 .

We make constant use of the notions and theorems contained in the book by one of us (C. Chevalley, Theory of Lie groups, I, Princeton University Press, 1940); this book will be referred to as LG.

\section{Chapter I. Manifolds With OpERATORS}

1. Differential forms on a manifold. Let $M$ be a manifold of dimension $d$ and class $C^{2}$. At every point $m$ of $M$ we consider the space $V(m)$ of tangent vectors to $M$ at $m$ [LG, p. 76] $\left(^{(}\right) . V(m)$ is a vector space of dimension $d$ over

(4) J. H. C. Whitehead, On the decomposition of an infinitesimal group, Proc. Cambridge Philos. Soc. vol. 32 (1936) pp. 229-237. Cf. also G. Hochschild, Semi-simple algebras and generalized derivations, Amer. J. Math. vol. 64 (1942) pp. 667-694.

(5) I. Ado, Über die Structur der endlichen kontinuierlichen Gruppen, Bull. Soc. Phys.-Math. Kazan (3) vol. 6 (1934) pp. 38-42. (Russian with German summary.)

( ${ }^{6}$ In LG, only analytic manifolds were considered. The definitions can be slightly modified in order to allow us to treat the case of manifolds of class $C^{k}(k \geqq 1)$. These modifications are trivial except as regards the definition of tangent vectors. This definition should be formulated as follows in the case of manifolds $M$ of class $C^{k}$. Let $m$ be any point of $M$ and let $A$ be the class 
the field $R$ of real numbers. Given an arbitrary finite-dimensional vector space $V$ over $R$, we denote by $C^{q}(m, V)$ the vector space of all $q$-linear alternating functions defined on $V(m)$ with values in $V$. If $V=R$ then $C^{q}(m)$ $=C^{q}(m, R)$ is the set of homogenous elements of order $q$ in the (contravariant) Grassmann algebra of $V(m)$. By definition $C^{0}(m, V)=V$.

A $V$-differential form (or shorter: $V$-form) of order $q$ on $M$ is a function $\omega$ which to each $m \in M$ assigns an element $\omega(m) \in C^{q}(m, V)$. If $V=R$ we omit the prefix $V$ - and speak of differential forms on $M$.

The usual definition of the differential $d \omega$ of a form of class $C^{1}$ [LG, p. 148 ] can be carried over to $V$-forms in the following manner. We select a basis $v_{1}, \cdots, v_{k}$ for the vectors in $V$. The $V$-form $\omega$ can then be written as $\omega=\omega^{(1)} v_{1}+\cdots+\omega^{(r)} v_{k}$ where $\omega^{(k)}$ are differential forms. Define $d \omega=d \omega^{(1)} v_{1}$ $+\cdots+d \omega^{(r)} v_{k}$. Clearly $d \omega$ is a $V$-form of order $q+1$ independent of the choice of the basis.

A $V$-form $\omega$ will be called regular if both $\omega$ and $d \omega$ are of class $C^{1}$. In the sequel all forms will be assumed regular without explicit statement. If $\omega$ is regular then (as a consequence of Stokes' formula) $d d \omega=0$, hence $d \omega$ also is regular.

$V$-forms $\omega$ such that $d \omega=0$ are called closed. Those of the form $\omega=d \theta$ where $\theta$ is a $V$-form of one lower order are called exact. Since $d d \omega=0$, every exact $V_{\mathrm{r}}$ form is closed. The quotient space of the tinear set of closed $V$-forms of order $q$ by the subspace of the exact $V$-forms of order $q$ will be denoted by $D^{q}(M, V)$ and by $D^{q}(M)$ if $V=R$. By analogy with topology $D^{q}(M, V)$ will be called the $q$-dimensional cohomology group of $M$ obtained using $V$-forms.

If $V=R$ then we also have the Grassmann multiplication of differential forms which to two forms $\omega^{p}$ and $\omega^{q}$ of order $p$ and $q$ respectively defines a form $\omega^{p} \square \omega^{q}$ of order $p+q$. This multiplication has the property [LG, p. 148] that

$$
d\left(\omega^{p} \square \omega^{q}\right)=d \omega^{p} \square \omega^{q}+(-1)^{p} \omega^{p} \square d \omega^{q}
$$

which implies that the product of two closed forms is closed and that the product of a closed form and of an exact one (taken in any order) is exact. Thus the Grassmann multiplication defines a multiplication of elements in $D^{p}(M)$ and $D^{q}(M)$ with values in $D^{p+q}(M)$. The direct sum $D(M)$ of the groups $D^{q}(M)$ for all $q \geqq 0$ thus becomes a ring (or rather an algebra over $R$ ) which we shall call the cohomology ring of $M$ obtained using differential forms.

Let $P: V \rightarrow V$ be a linear transformation. For every $f \in C^{q}(m, V)$ we then

of functions of type $C^{k}$ at $m$. A tangent vector $L$ to $M$ at $m$ is a linear real-valued function defined on $A$ satisfying the following condition: let $f_{1}, \cdots, f_{r} \in A$ and let $\phi\left(u_{1} \cdots, u_{r}\right)$ be a reai-valued function of $r$ real arguments, which is of type $C^{k}$ in the neighborhood of $\left(a_{1}, \cdots, a_{r}\right)$ where $a_{i}=f_{i}(m)(1 \leqq i \leqq r)$. If $f \in A$ can be expressed in the neighborhood of $m$ as $f=\phi\left(f_{1}, \cdots, f_{r}\right)$ then $L f=\sum_{i=1}^{r}\left(\partial \phi / \partial u_{i}\right)\left(a_{1}, \cdots, a_{r}\right) L f_{i}$. 
have the composite function $P f \in C^{q}(m, V)$ and the correspondence $f \rightarrow P f$ is a linear transformation $P: C^{q}(m, V) \rightarrow C^{q}(m, V)$. Hence for each $V$-form $\omega$ on $M$ we may define a $V$-form $P \omega$ by setting $(P \omega) m=P(\omega m)$. It is easy to see that $P \omega$ is regular if $\omega$ is and that

$$
\begin{aligned}
& d(P \omega)=P d \omega, \\
& P_{1}\left(P_{2} \omega\right)=\left(P_{1} P_{2}\right) \omega, \\
& P\left(r_{1} \omega_{1}+r_{2} \omega_{2}\right)=r_{1} P \omega_{1}+r_{2} P \omega_{2} \text {, } \\
& r_{1}, r_{2} \in R \text {. }
\end{aligned}
$$

Consider two manifolds $M_{1}, M_{2}$, a transformation $T: M_{1} \rightarrow M_{2}$ (all of class $C^{2}$ ) and a $V$-form $\omega$ on $M_{2}$. If $m \in M_{1}$ then $T$ defines [LG, p. 78] a linear mapping of the tangent vector spaces $d T: V(m) \rightarrow V(T m)$. If $f$ is any $q$-linear function on $V(T m)$ then $f T$ defined by

$$
(f T)\left(x_{1}, \cdots, x_{q}\right)=f\left(d T x_{1}, \cdots, d T x_{q}\right), \quad x_{1}, \cdots, x_{q} \in V(m),
$$

is a $q$-linear function on $V(m)$. Using this notation we define a $V$-form $\omega T$ on $M_{1}$ by setting

$$
(\omega T) m=\omega(T m) T .
$$

If $\omega$ is regular then so is $\omega T$. The following properties of $\omega T$ will be used [LG, p. 152]:

$$
\begin{aligned}
d(\omega T) & =(d \omega) T, \\
\left(r_{1} \omega_{1}+r_{2} \omega_{2}\right) T & =r_{1}\left(\omega_{1} T\right)+r_{2}\left(\omega_{2} T\right), \\
P(\omega T) & =(P \omega) T, \\
\left(\omega T_{2}\right) T_{1} & =\omega\left(T_{2} T_{1}\right),
\end{aligned}
$$

where, in (1.8), $T_{1}$ is a mapping of class $C^{2}$ of $M_{1}$ into $M_{2}$ and $T_{2}$ a mapping of class $C^{2}$ of $M_{2}$ into some third manifold $M_{3}$ of class $C^{2} ; \omega$ is a form on $M_{3}$.

2. Equivariant forms. We shall assume that a topological group $G$ is acting as a group of transformations on $M$. By this we mean that for each $g \in G$ a. transformation

$$
T_{0}: M \rightarrow M
$$

of class $C^{2}$ is given such that

$T_{\theta}(m)$ is continuous in $g$ and $m$ simultaneousl $y$,

$$
\begin{aligned}
T_{o_{1} o_{2}} & =T_{o_{1}} T_{o_{2}}, & \\
T_{\bullet}(m) & =m & \text { for each } m \in M,
\end{aligned}
$$

where $e$ denotes the unit element of $G$.

We shall also assume that a representation $P$ of $G$ with a finite-dimensional vector space $V$ over $R$ as representation space is given. For each $g \in G$ a 
linear transformation $P_{\ominus}: V \rightarrow V$ is then defined satisfying conditions analogous to (2.1)-(2.3).

A (regular) $V$-form $\omega$ on $M$ will be called equivariant provided

$$
P_{0} \omega=\omega T_{0}
$$

for each $g \in G$. It follows from (1.2) and (1.5) that if $\omega$ is equivariant then $d \omega$ is equivariant. Propositions (1.4) and (1.6) imply that if $\omega_{1}$ and $\omega_{2}$ are equivariant, so is $r_{1} \omega_{1}+r_{2} \omega_{2}$ for any $r_{1}, r_{2} \in R$.

A $V$-form which is the differential of an equivariant form will be called equivariantly exact. The quotient space of the linear set of closed equivariant $V$-forms of order $q$ on $M$ by the subset of equivariantly exact $V$-forms will be denoted by $E^{q}(M, P)$. We shall refer to $E^{q}(M, P)$ as the $q$-dimensional cohomology group of $M$ obtained using equivariant $V$-forms.

If $V=R$ and $P$ is the trivial representation we shall write $E^{q}(M)$ instead of $E^{q}(M, P)$. Since the Grassmann product of two equivariant forms is equivariant, it follows as before that the direct sum of the spaces $E^{q}(M)$ forms a ring $E(M)$.

The equivariant $V$-forms form a linear subspace of the space of $V$-forms. This leads to a natural homomorphism of the cohomology groups

$$
\pi: E^{q}(M, P) \rightarrow D^{q}(M, V) .
$$

If $V=R$ and $P$ is trivial, $\pi$ is a ring homomorphism.

The following theorem will be proved in $\$ 6$.

THEOREM 2.1. If $G$ is compact then (2.5) maps $E^{q}(M, P)$ isomorphically into a subspace of $D^{q}(M, V)$.

A more detailed analysis of (2.5) follows from the decomposition of $P$ into irreducible components. Such a decomposition always exists if $G$ is compact. Let then $V=V_{1}+\cdots+V_{k}$ be a direct decomposition of $V$ into irreducible invariant subspaces and let $P_{i}$ be the corresponding representations of $G$ in $V_{i}$. Every $V$-form $\omega$ then decomposes uniquely as

$$
\omega=\omega_{1}+\cdots+\omega_{k}
$$

with $\omega_{i}$ being a $V_{i}$-form. There result direct sum decompositions

$$
E^{q}(M, P)=\sum_{i} E^{q}\left(M, P_{i}\right), \quad D^{q}(M, V)=\sum_{i} D^{q}\left(M, V_{i}\right)
$$

and an appropriate decomposition of (2.5). Hence we may concentrate our attention on irreducible representations.

In the following two theorems it is assumed that $G$ is compact and connected. The proofs will be given in $\$ 6$.

THEOREM 2.2. If the representation $P$ of $G$ is irreducible and nontrivial 
then $E^{q}(M, P)=\{0\}$, that is, every closed equivariant $V$-form is equivariantly exact.

THEOREM 2.3. If $V=R$ and $P$ is trivial, the correspondence (2.5) is a ring isomorphism onto

$$
E(M) \approx D(M),
$$

that is, the equivariant forms lead to the same cohomology ring as all the regular differential forms on $M$.

3. The averaging process. We assume that $G$ is compact. This implies the existence of a Haar measure with the measure of $G$ being 1 . The definition and properties of integrals of real valued functions defined on $G$ carry over in a trivial fashion to functions with values in a finite-dimensional vector space $V$ over $R$

Given a continuous $V$-form $\omega$ of order $q$ on $M$ consider the family of $V$ forms

$$
\omega^{\theta}=P_{\theta^{-1} \omega} T_{\theta},
$$

For each $m \in M, \omega^{o}(m)$ is a continuous function on $G$ with values in the vector space $C^{q}(m, V)$ (this will be established in the course of the proof of (3.1) and (3.2) below). Hence the integral

$$
(I \omega) m=\int_{G} \omega^{o}(m) d g
$$

is a well defined element of $C^{q}(m, V)$. The $V$-form $I \omega$ thus obtained has the following properties (for $\omega$ regular)

$I \omega$ is regular,

$$
d(I \omega)=I(d \omega),
$$

$I \omega$ is equivariant,

if $\omega$ is equivariant then $I \omega=\omega$.

Proof of (3.1) and (3.2). Having selected a base in the vector space $V$, we can represent $\omega$ by its components $\omega^{(k)}$ with respect to this base; each $\omega^{(k)}$ is then a differential form of class $C^{1}$ on $M$. Let $m_{0} \in M$ and $g_{0} \in G$; we select coordinate systems $\left(x_{1}, \cdots, x_{n}\right)$ and $\left(y_{1}, \cdots, y_{n}\right)$ on $M$ at $m_{0}$ and $T_{0_{0}}\left(m_{0}\right)$ respectively. We can find a cubic neighbourhood $U=U\left(m_{0}, g_{0}\right)$ of $m_{0}$ with respect to $\left(x_{1}, \cdots, x_{n}\right)$, a cubic neighbourhood $W$ of $T_{\theta_{0}}\left(m_{0}\right)$ with respect to $\left(y_{1}, \cdots, y_{n}\right)$ and a neighbourhood $N=N\left(m_{0}, g_{0}\right)$ of $g_{0}$ in $G$ such that $T_{\theta}(U) \subset W$ for all $g \in N$. In $W$ each $\omega^{(k)}$ has a certain analytic expression

$$
\omega^{(k)}=\sum_{i_{1}, \cdots, i_{q}} A_{i_{1}}^{(k)} \cdots, i_{q}\left(y_{1}, \cdots, y_{n}\right) d y_{i_{1}} \cdots d y_{i_{q}}
$$


with respect to the coordinates $y_{1}, \cdots, y_{n}$; the functions $A_{i_{1}, \ldots, l_{n}}^{(k)}$ are of class $C^{1}$. On the other hand if $m \in U$ is a point with coordinates $\left(x_{1}, \cdots, x_{n}\right)$, and if $g \in N$, the $y$-coordinates of $T_{\vartheta}(m)$ are functions

$$
y_{i}=\theta_{i}\left(x_{1}, \cdots, x_{n} ; g\right)
$$

of $x_{1}, \cdots, x_{n} ; g$. For $g$ fixed, $\theta_{i}$ are of class $C^{2}$ in $x_{1}, \cdots, x_{n}$. We have

$$
\left(\omega^{(k)} T_{g}\right)(m)=\sum_{i_{1}, \cdots, i_{q}} A_{i_{1}, \cdots, i_{q}}^{(k)}\left(\theta_{1}, \cdots, \theta_{n}\right) d \theta_{i_{1}} \cdots d \theta_{i_{q}} .
$$

On the other hand the mapping $P_{a}$ can be represented (with respect to the base in $V$ ) by a matrix $\rho_{k l}(g)$, and we have

$$
\left(\omega^{\sigma}(m)\right)^{(k)}=\sum_{\imath \rho_{k l}}\left(g^{-1}\right) \sum_{i_{1}, \cdots, i_{q}} A_{i_{1}}^{(l)} \cdots, i_{q}\left(\theta_{i_{1}}, \cdots, \theta_{n}\right) d \theta_{i_{1}} \cdots d \theta_{i_{q}} .
$$

Now, it follows from a theorem of Montgomery $\left({ }^{7}\right)$ that not only the functions $\theta_{i}$ but also their partial derivatives of order 1 and 2 are continuous functions of $\left(x_{1}, \cdots, x_{n} ; g\right)$. This implies that

$$
\left(\omega^{o}(m)\right)^{(k)}=\sum_{i_{1}, \cdots, i_{q}} B_{i_{1}}^{(k)} \cdots, i_{q}\left(x_{1}, \cdots, x_{n} ; g\right) d x_{i_{1}} \cdots d x_{i_{q}}
$$

where each $B_{i_{1}}^{(k)}, \ldots, \imath_{q}$ for $g$ fixed is of class $C^{1}$ and is continuous, together with its partial derivatives in $\left(x_{1}, \cdots, x_{n} ; g\right)$.

Let $A$ be any measurable subset of $N\left(m_{0}, g_{0}\right)$. Then

$$
\left(\int_{A} \omega^{g} d g\right)^{(k)}(m)=\sum_{i_{1} \cdots i_{q}}\left(\int_{A} B_{i_{1} \cdots i_{q}}^{(k)}\left(x_{1}, \cdots, x_{n} ; g\right) d g\right) d x_{i_{1}} \cdots d x_{i_{q}}
$$

and the functions $\int_{A} B_{i_{1}}^{(k)} \ldots, s_{q}\left(x_{1}, \cdots, x_{n} ; g\right) d g$ are of class $C_{1}$. Consequently $\int_{A} \omega^{0} d g$ is a $V$-form on $M$ of class $C^{1}$ on $U\left(m_{0}, g_{0}\right)$.

Moreover, we have

$$
\frac{\partial}{\partial x_{i_{0}}} \int_{A} B_{i_{1}}^{(k)} \cdots, i_{q}\left(x_{1}, \cdots, x_{n}, g\right) d g=\int_{A} \frac{\partial}{\partial x_{i_{0}}} B_{i_{1}}^{(k)} \cdots, i_{q}\left(x_{1}, \cdots, x_{n}, g\right) d g .
$$

Therefore

$$
\left(d \int_{A} \omega^{\sigma} d g\right)(m)=\left(\int_{A} d \omega^{\sigma} d g\right)(m)=\left(\int_{A}(d \omega)^{o} d g\right)(m)
$$

for $m \in U\left(m_{0}, g_{0}\right)$.

Keeping $m_{0}$ fixed we now vary $g_{0}$. Since $G$ is compact, there is a finite sequence $g_{i}, \cdots, g_{\text {e }}$ such that the neighbourhoods $N\left(m_{0}, g_{i}\right)$ cover $G$. Let $U\left(m_{0}\right)=\bigcap_{i} U\left(m_{0}, g_{i}\right)$. It follows that $\int_{G^{0}} \omega^{0} d g$ is of class $C^{1}$ on $U\left(m_{0}\right)$ and that $d\left(\int_{G} \omega^{0} d g\right)(m)=\left(\int_{G}(d \omega)^{\circ} d g\right)(m)$ for $m \in U\left(m_{0}\right)$. Hence $I \omega$ is of class $C_{1}$ and

(7) D. Montgomery, Topological groups of differentiable transformations, Ann. of Math. vol. 46 (1945) pp. 382-387; Theorem 1, p. 383 and Corollary 1, p. 386. 
$d(I \omega)=I(d \omega)$. Consequently $d(I \omega)$ also is of class $C^{1}$ and $I \omega$ is regular.

Proof of (3.3). For every $h \in G$ and $m \in M$, we have

$$
\begin{aligned}
{\left[(I \omega) T_{h}\right](m) } & =I \omega\left(T_{h} m\right) T_{h}=\left(\int \omega^{o}\left(T_{h} m\right) d g\right) T_{h} \\
& =\int \omega^{o}\left(T_{h} m\right) T_{h} d g=\int\left(\omega^{o} T_{h}\right)(m) d g=\int\left(P_{g}{ }^{-1} \omega^{o} T_{o} T_{h}\right)(m) d g \\
& =\int\left(P_{h} \omega^{o h}\right)(m) d g=P_{h} \int \omega^{o h}(m) d g=P_{h} \int \omega^{o}(m) d g \\
& =\left[P_{h} I(\omega)\right](m) .
\end{aligned}
$$

Hence $(I \omega) T_{h}=P_{h} I(\omega)$.

Proof of (3.4). If $\omega$ is equivariant then $\omega^{g}=\omega$ for all $g \in G$ and $(I \omega)(m)$ $=\int_{G} \omega(m) d g=\omega(m)$ since $G$ has measure 1.

4. Integration of forms over cycles. We shall use the singular homology theory as developed by one of the authors $\left.{ }^{8}\right)$. In particular $S(M)$ will denote the singular complex of the manifold $M$. The cells of $S(M)$ are equivalence classes of singular simplexes $T$ which are continuous maps into $M$

$$
T: s \rightarrow M
$$

of euclidean simplexes $s$ with ordered vertices.

Suppose now that the manifold $M$ is of class $C^{k}$. If the mapping $T$ can be extended to a neighborhood $U_{s}$ of $s$ (in the cartesian space containing $s$ ) in such a way that the extended map $T^{\prime}$ be of class $C^{k}$, then we shall say that $T$ is a singular simplex of class $C^{k}$. The singular simplexes of class $C^{k}$ form a closed subcomplex $S^{k}(M)$ of $S(M)$, and we have the identity chain transformation

$$
\epsilon^{k}: S^{k}(M) \rightarrow S(M) \text {. }
$$

It has been proved $\left({ }^{9}\right)$ that $\epsilon^{k}$ induces isomorphisms of the respective homology and cohomology groups.

We now return to our assumption that $M$ is of class $C^{2}$ and we shall consider only chains ard cycles in $S^{2}(M)$. Let

$$
T: s \rightarrow M
$$

be a singular $q$-dimensional simplex of class $C^{2}$. We shall assume that $T$ has been extended to a map $T^{\prime}$ of class $C^{2}$ of some neighbourhood $U_{s}$ of $s$. Given a continuous differential form $\omega$ of order $q$ on $M$ we then have the form $\omega T^{\prime}$ on $U_{s}$ and the integral $\int_{8} \omega T^{\prime}$ is defined in the usual way. This integral is inde-

(8) S. Eilenberg, Singular homology theory, Ann. of Math. vol. 45 (1944) pp. 407-447.

( $)$ S. Eilenberg, Singular homology in differentiable manifolds, Ann. of Math. vol. 48 (1947) pp. $670-681$. 
pendent of the choice of the extension $T^{\prime}$ and of the choice of $T$ within its equivalence class.

We define

$$
\int_{T} \omega=\int_{s} \omega T^{\prime}
$$

Given a $q$-dimensional chain $c=\sum r_{i} T_{i}$ in $S^{2}(M)$ (coefficients in $R$ ) we define

$$
\int_{c} \omega=\sum r_{i} \int_{T_{i}} \omega
$$

If $\omega$ is of class $C^{1}$ then $\omega T^{\prime}$ is of class $C^{1}$ and the classical Stokes formula implies that

$$
\int_{c} d \omega=\int_{\partial c} \omega
$$

for every $(q+1)$-chain $c$. Hence if $\omega$ is closed then $\int_{\partial c} \omega=0$. It follows that for every homology class $z$ of dimension $q$ and every closed form $\omega$ of order $q$ and class $C^{1}$ the integral $\int_{2} \omega$ is defined without ambiguity.

Let $\omega$ be a closed $V$-form of class $C^{1}$. Taking a base in $V$, denote by $\omega^{(k)}$ the components of $\omega$ with respect to this base and by $\int_{x} \omega$ the element of $V$ whose components are the real numbers $\int_{z} \omega^{(k)}$. Clearly $\int_{x} \omega$ is independent of the choice of base in $V$.

If $P: V \rightarrow V$ is a linear transformation then clearly

$$
\int_{z} P \omega=P\left(\int_{z} \omega\right)
$$

Let two manifolds $M_{1}, M_{2}$ and a mapping $R: M_{1} \rightarrow M_{2}$, all of class $C^{2}$, be given. Let $\omega$ be a $V$-form on $M_{2}$ of order $q$ and class $C^{1}$ and let $T: s \rightarrow M_{1}$ be a singular $q$-simplex of class $C^{1}$. With $T^{\prime}$ defined as before, we have $(\omega R) T^{\prime}$ $=\omega\left(R T^{\prime}\right)$. Therefore

$$
\int_{T} \omega R=\int_{s} \omega R T^{\prime}=\int_{R T} \omega
$$

where $R T: s \rightarrow M_{2}$ is a singular simplex of $M_{2}$. Consequently for every closed form $\omega$ on $M_{2}$

$$
\int_{z} \omega R=\int_{R z} \omega
$$

where $R z$ is the image of the homology class $z$ under the homomorphism of the homology groups induced by $R$.

5. Formulation of de Rham's theorems. Let $H_{q}(M)$ and $H^{q}(M)$ denote 
the $q$-dimensional homology and cohomology groups of $M$ with real coefficients defined using the complex $S(M)$. The groups $H^{q}(M)$ and $H_{q}(M)$ are in duality, the product of a cohomology class $f$ and a homology class $z$ being the Kronecker index $K I(f, z)$.

For a fixed closed differential form $\omega$ of order $q$ on $M$ the expression $\int_{z} \omega$ is a linear function on $H_{q}(M)$, hence there is a unique cohomology class $f \in H^{q}(M)$ such that

$$
\int_{z} \omega=K I(f, z)
$$

for each $z \in H_{q}(M)$. De Rham's theorems $\left(^{(8)}\right.$ imply that the correspondence $\omega \rightarrow f$ establishes an isomorphism.

$$
D^{q}(M) \approx H^{q}(M) .
$$

Moreover, this isomorphism is a ring isomorphism of the cohomology ring $D(M)$ obtained using differential forms (with the Grassmann multiplication) with the cohomology ring $H(M)$ (with the cup product as multiplication).

In the next section we shall use the following part of the previously stated theorem :

(5.1) If $\omega$ is a closed form such that $\int_{z} \omega=0$ for every homology class, then $\omega$ is exact.

There is no explicit proof of the above theorem in the literature. De Rham's original proof is valid for closed manifolds $M$ carrying a simplicial decomposition of a rather special kind $\left({ }^{10}\right)$.

Theorems 2.2 and 2.3 combined with the theorem of de Rham imply that if $G$ is compact and connected then both $D^{q}(M, V)$ and $E^{q}(M, P)$ are isomorphic with certain multiples (in the sense of direct sum) of the cohomology group $H^{q}(M)$ (real coefficients). In the case of $D^{q}$ the multiplicity is the dimension of $V$, in the case of $E^{a}$ it is the number of times the trivial representation occurs in the irreducible decomposition of the representation $P$.

6. Proofs of Theorems 2.1-2.3. To prove Theorem 2.1 consider an equivariant closed $V$-form $\omega$ which is exact. Then $\omega=d \theta$ for some $V$-form $\theta$. By (3.2) and (3.4) we have

$$
d(I \theta)=I(d \theta)=I \omega=\omega .
$$

Since $I \theta$ is equivariant by (3.3), it follows that $\omega$ is equivariantly exact.

Before we proceed with the proofs of Theorems 2.2 and 2.3 we prove the following proposition:

(6.1) If $G$ is connected then for every $g \in G$ and every homology class $z$ in $M, T_{g} z=z$.

(10) Cf. footnote 3, p. 62. 
Let $M$ be given as some simplicial decomposition and let $K$ be a subcomplex of $M$ containing a cycle of the homology class $z$. Let $K_{1}$ be a complex containing $K$ in its interior. We may then find a neighbourhood $U$ of the identity $e$ in $G$ such that $T_{g}(K) \subset K_{1}$ for each $g \in U$. If we consider the family of mappings $T_{0}: K \rightarrow K_{1}, g \in U$, it follows that there is a neighbourhood $U_{1} \subset U$ of the identity such that any map $T_{0}: K \rightarrow K_{1}$ with $g \in U_{1}$ is homotopic with the identity map $T_{e}: K \rightarrow K_{1}$. Hence $T_{0} z=z$ for $g \in U_{1}$. Since $G$ is connected, this holds for any $g \in G$.

Proof of Theorem 2.2. Let $\omega$ be a closed equivariant $V$-form on $M$. Since $P_{0} \omega=\omega T_{0}$ for each $g \in G$ it follows from (4.1), (4.2) and (6.1) that for every homology class $z$

$$
P_{g} \int_{z} \omega=\int_{z} P_{\theta} \omega=\int_{z} \omega T_{g}=\int_{T_{g z}} \omega=\int_{z} \omega .
$$

Since this holds for every $g \in G$ and since the representation $P$ is irreducible and nontrivial it follows that $\int_{z} \omega=0$. Since this holds for every $z,(5.1)$ implies that $\omega$ is exact and, by Theorem $2.1, \omega$ is equivariantly exact.

Proof of Theorem 2.3. We have already shown that $\pi: E^{q}(M) \rightarrow D^{q}(M)$ is an isomorphism into. It is therefore sufficient to prove that $E^{q}(M)$ is mapped onto $D^{q}(M)$. Let $\omega$ be a closed form of order $q$ on $M$. Consider the integral $\int_{z} I \omega$ over a $q$-dimensional homology class $z$. We have

$$
\int_{z} I \omega=\int_{z} \int_{G} \omega^{o} d g=\int_{z} \int_{G} \omega T_{0} d g \text {. }
$$

Since all the functions involved are continuous, Fubini's theorem can be applied; reversing the order of integration and using (4.2) and (6.1) we have

$$
\int_{z} I \omega=\int_{G} \int_{z} \omega T_{\theta} d g=\int_{G} \int_{T_{\theta z}} \omega d g=\int_{G} \int_{z} \omega d g=\int_{z} \omega .
$$

Hence $\int_{z}(\omega-I \omega)=0$ and, by $(5.1), \omega-I \omega$ is exact. This completes the proof.

7. Double equivariance. Let $G$ and $H$ be two groups operating on $M$. We shall assume here that $V=R$. A regular differential form $\omega$ on $M$ will be called doubly equivariant provided

$$
\omega T_{o}=\omega=\omega T_{h}
$$

for all $g \in G$ and $h \in H$. As before we may define cohomology groups $E^{q}(M)$ using doubly equivariant forms only, and the cohomology ring $\tilde{E}(M)$. As before we have a natural ring homomorphism

$$
\pi: E(M) \rightarrow D(M) .
$$

THEOREM 7.1. If $G$ and $H$ are compact and connected and if the transformations $T_{0}$ and $T_{h}$ commute, 


$$
T_{o} T_{h}=T_{h} T_{。}
$$

for all $g \in G, h \in H$, then (7.1) is a ring isomorphism onto

$$
\tilde{E}(M) \approx D(M) .
$$

Proof. Consider the direct product $G \times H$. For $(g, h) \in G \times H$ define $T_{(g, h)}=T_{0} T_{h}$. It follows from our assumptions that $G \times H$ is a compact and connected group operating on $M$. Let $\omega$ be a form equivariant relative to $G \times H$, then $\omega T_{0} T_{h}=\omega$. Taking $g=e_{G}$ we find $\omega T_{h}=\omega$ and similarly $\omega T_{0}=\omega$ so that $\omega$ is doubly equivariant. Conversely every doubly equivariant form is equivariant relative to $G \times H$. Thus Theorem 7.1 is a consequence of Theorem 2.3.

\section{Chapter II. Localization}

8. The transitive case. We shall assume now that the compact group $G$ operates on the connected manifold $M$ transitively, that is, that for each pair $m_{1}, m_{2} \in M$ there is an element $g$ in $G$ such that $T_{o} m_{1}=m_{2}$. We further assume that $G$ operates on $M$ effectively, that is; that none of the transformations $T_{g}$, except $T_{e}$, leave all the points of $M$ fixed.

Let $m_{0}$ be a point of $M$, and let $H$ be the group of elements $h \in G$ such that $T_{h} m_{0}=m_{0}$. Then for any $g \in G, T_{o} m_{0}$ depends only on the coset $g H$ of $g$ modulo $H$. The mapping $g H \rightarrow T_{\imath} m_{0}$ is then a 1-1 continuous mapping of $G / H$ onto $M$, and since $G / H$ is compact, it is a homeomorphism. On the other hand it follows from a theorem of Montgomery $\left.{ }^{11}\right)$ that $G$ is in this case a Lie group; therefore $G / H$ admits the structure of an analytic manifold. Bochner and Montgomery ${ }^{(2)}$ have also proved that the mapping $g H \rightarrow T_{0} m_{0}$ and its inverse are both of class $C^{2}$. Therefore, we may assume without loss of generality that $M$ is identical with $G / H$ and that $T_{g_{1}}\left(g_{2} H\right)=\left(g_{1} g_{2}\right) H$.

This being done, the assumption that $G$ is compact is no longer needed. In the remainder of this chapter $G$ will be an arbitrary Lie group and $H$ a closed subgroup of $G$.

For the moment we shall study the simple case when $H$ is the trivial subgroup and $M=G$. We shall return to the case of a nontrivial $H$ at the end of this chapter.

Given any function $f$ defined on $G$ it will be convenient to denote by $f_{e}$ the value of $f$ at the unit element $e$ of $G$.

Let $L$ be the Lie algebra of the group $G$ [LG, p. 101]. The elements of $L$ are left invariant infinitesimal transformations $x$ of $G$. Hence $x_{e}$ is an element of the tangent vector space $V_{e}=V(e)$ to $G$ at $e$. Because of the left invariance condition, $x$ is entirely determined by $x_{e}$, and we may therefore regard $V_{e}$ as the vector space of the Lie algebra $L$.

(11) Cf. footnote 7, Theorem 2, p. 387.

(12) S. Bochner and D. Montgomery, Groups of differentiable and real or complex analytic transformations, Ann. of Math. vol. 46 (1945) pp. 685-694. 
Given any $V$-form $\omega$ of order $q$ on $G$, the element $\omega_{e}$ of $C^{q}(1, V)$ is a $q$-linear alternating function on $V_{e}$ to $V$. We define

$$
\{\omega\}\left(x_{1}, \cdots, x_{q}\right)=\omega_{e}\left(x_{1 e}, \cdots, x_{q e}\right), \quad x_{i} \in L .
$$

This way to each $V$-form $\omega$ of order $q$ on $G$ there corresponds a $q$-linear alternating function $\{\omega\}$ with arguments in the Lie algebra $L$ and values in the vector space $V$. The correspondence $\omega \rightarrow\{\omega\}$ is obviously linear. Moreover if $\omega$ is equivariant then $\{\omega\}=0$ implies $\omega=0$. The last fact follows from the remark that the condition $P_{h} \omega=\omega T_{h}$ implies that $\omega(\mathrm{g})$ can be obtained from $\omega_{\bullet}$ by means of suitable linear transformations, and hence $\omega_{e}=0$ implies $\omega=0$.

The passage from $\omega$ to $\{\omega\}$ will be referred to as localization.

9. Localization of left invariant forms. We shall assume that $V=R$ and that the representation $P$ of $G$ in $V$ is trivial. The equivariance condition on a differential form then becomes a condition of left invariance: $\omega=\omega T_{h}$ where $T_{h} g=h g$. We consider two Grassmann algebras: $1^{\circ}$ the algebra of left invariant differential forms on $G, 2^{\circ}$ the (contravariant) Grassmann algebra of the vector space of the Lie algebra $L$. The correspondence $\omega \rightarrow\{\omega\}$ is then multiplicative:

$$
\left\{\omega_{1} \square \omega_{2}\right\}=\left\{\omega_{1}\right\} \square\left\{\omega_{2}\right\} .
$$

THeоReм 9.1. Let $G$ be a Lie group. The correspondence $\omega \rightarrow\{\omega\}$ establishes an isomorphism between the algebra of left invariant differential forms on $G$ and the (contravariant) Grassmann algebra of the vector space of the Lie algebra $L$ of $G$. Moreover

$$
\begin{aligned}
& \{d \omega\}\left(x_{1}, \cdots, x_{q+1}\right) \\
& =\frac{1}{q+1} \sum_{k<l}(-1)^{k+l+1}\{\omega\}\left(\left[x_{k}, x_{l}\right], x_{1}, \cdots, \hat{x}_{k}, \cdots, \hat{x}_{l}, \cdots, x_{q+1}\right) .
\end{aligned}
$$

The symbol over a variable indicates that the appropriate variable is to be omitted.

Proof. Let $f$ be a $q$-linear alternating function on the Lie algebra $L$. Represent $f$ as a finite sum of Grassmann products

$$
f^{k}=f_{1}^{k} \square \cdots \square f_{q}^{k}
$$

where $f_{i}^{k}$ is a linear function on $L$. For each $f_{i}^{k}$ there is [LG, p. 152] an analytic left invariant form $\omega_{i}^{k}$ of order 1 on $G$ such that $\left\{\omega_{i}^{k}\right\}=f_{i}^{k}$. Define $\omega^{k}=\omega_{1}^{k}$ $\square \cdots \square \omega_{a}^{k}$. Clearly $\omega^{k}$ is analytic and left invariant and by $(9.1)\left\{\omega^{k}\right\}=f^{k}$. This proves the first half of theorem, and in addition it proves that every left-invariant form is analytic.

Before we proceed with the proof of (9.2) it will be convenient to give this expression a slightly different form. We first notice that the right-hand side of (9.2) is an alternating function of the variables $x_{1}, \cdots, x_{q+1}$. Hence if 
we apply the operation of alternation, the right-hand side will not change its value. On the other hand the right-hand side of (9.2) is the sum of $q(q+1) / 2$ terms each of which is obtained from the term

$$
\{\omega\}\left(\left[x_{1}, x_{2}\right], x_{3}, \cdots, x_{q+1}\right)
$$

by applying certain permutations to the variables and multiplying by \pm 1 according to whether the permutation was even or odd. Hence applying the operation of alternation to the various summands in (9.2) will each time give the same result as alternating (9.3). Hence (9.2) is equivalent with the following formula,

$$
\{d \omega\}\left(x_{1}, \cdots, x_{q+1}\right)=\frac{q}{2} A\left[\{\omega\}\left(\left[x_{1}, x_{2}\right], x_{3}, \cdots, x_{q+1}\right)\right]
$$

where $A$ is the abbreviation for "alternation."

We prove (9.4) by induction on $q$. For $q=1,(9.4)$ reads

$$
\{d \omega\}\left(x_{1}, x_{2}\right)=\{\omega\}\left(\left[x_{1}, x_{2}\right]\right) / 2 .
$$

This formula for left invariant differential forms $\omega$ of order 1 is known [LG, p. 153].

Assume that (9.4) holds for orders less than $q$. Let

$$
\omega=\omega_{1} \square \omega_{2}
$$

be a left invariant form of order $q$ which is a Grassmann product of a left invariant form $\omega_{1}$ of order 1 and a left invariant form $\omega_{2}$ of order $q-1$. Since

$$
d \omega=d \omega_{1} \square \omega_{2}-\omega_{1} \square d \omega_{2}
$$

we have, by the inductive hypothesis,

$$
\begin{aligned}
\{d \omega\}\left(x_{1}, \cdots, x_{q+1}\right)= & \frac{1}{2} A\left[\left\{\omega_{1}\right\}\left(\left[x_{1}, x_{2}\right]\right)\left\{\omega_{2}\right\}\left(x_{3}, \cdots, x_{q+1}\right)\right] \\
& -\frac{q-1}{2} A\left[\left\{\omega_{1}\right\}\left(x_{1}\right)\left\{\omega_{2}\right\}\left(\left[x_{2}, x_{3}\right], x_{4}, \cdots, x_{q+1}\right)\right] \\
= & \frac{1}{2} A\left[\{\omega\}\left(\left[x_{1}, x_{2}\right], x_{3}, \cdots, x_{q+1}\right)\right] \\
& -\frac{q-1}{2} A\left[\{\omega\}\left(x_{1},\left[x_{2}, x_{3}\right], x_{4}, \cdots, x_{q+1}\right)\right] \\
= & \frac{q}{2} A\left[\{\omega\}\left(\left[x_{1}, x_{2}\right], x_{3}, \cdots, x_{q+1}\right)\right] .
\end{aligned}
$$

10. Localization of equivariant $V$-forms. Let $P$ be a representation of the Lie group $G$ by means of linear transformations of the $n$-dimensional vector space $V$. Having chosen basis vectors $v_{1}, \cdots, v_{n}$ in $V$ each linear 
transformation $P_{0}$ is described by a matrix $\left|a_{i j}(g)\right|$. The coefficients $a_{i j}(g)$ are analytic functions of $g$, and their differentials $d a_{i j}$ are analytic forms of order 1 on $G$. For every $x$ in the Lie algebra $L$ of $G$ one considers the matrix

$$
P(x)=\left|\left\{d a_{i j}\right\}(x)\right|
$$

which again may be regarded as a linear transformation of $V$ into itself. The mapping $x \rightarrow P(x)$ is a representation of the Lie algebra $L$.

THEOREM 10.1. Let $G$ be a Lie group and $P$ a representation of $G$ by means of linear transformations of a finite-dimensional vector space $V$ over the field of real numbers. The correspondence $\omega \rightarrow\{\omega\}$ establishes an isomorphism of the space of equivariant $V$-forms $\omega$ of order $q$ on $G$ and the space of $q$-linear alternating functions defined on the Lie algebra $L$ of $G$ with values in $V$. Moreover

$$
\begin{aligned}
& \{d \omega\}\left(x_{1}, \cdots, x_{q+1}\right) \\
& =\frac{1}{q+1} \sum_{k<l}(-1)^{k+l+1}\{\omega\}\left(\left[x_{k}, x_{l}\right], x_{1}, \cdots, \hat{x}_{k}, \cdots, \hat{x}_{l}, \cdots, x_{q+1}\right) \\
& \quad+\frac{1}{q+1} \sum_{k}(-1)^{k+1} P\left(x_{k}\right)\{\omega\}\left(x_{1}, \cdots, \hat{x}_{k}, \cdots, x_{q+1}\right) .
\end{aligned}
$$

Proof. Let $f$ be a $q$-linear alternating function on $L$ to $V$. In terms of the basis vectors $v_{1}, \cdots, v_{n}$ in $V, f$ can be written as

$$
f=f_{1} v_{1}+\cdots+f_{n} v_{n}
$$

where each $f_{i}$ is a $q$-linear alternating function on $L$ with real values. By Theorem 9.1 there exist left invariant analytic forms $\Omega_{i}$ of order $q$ on $G$ such that $\left\{\Omega_{i}\right\}=f_{i}$. Define the left invariant $V$-form $\Omega$ on $G$ by $\Omega=\Omega_{1} v_{1}+\ldots$ $+\Omega_{n} v_{n}$ then set

$$
\omega(g)=P_{g} \Omega(g)
$$

or more precisely

$$
\omega(g)=\sum_{i, j} a_{i j}(g) \Omega_{j}(g) v_{i} .
$$

Since the $a_{i j}(g)$ 's are analytic it follows that $\omega$ is an analytic $V$-form of order $q$ on $G$. Since $\omega_{e}=P_{e} \Omega_{e}=\Omega_{e}$ it follows that $\{\omega\}=\{\Omega\}=f$.

Let $g \in G$ and let $T_{0}: g^{\prime} \rightarrow g g^{\prime}$ be the corresponding left translation of $G$. Since $\Omega$ is left invariant we have

$$
\begin{aligned}
\left(\omega T_{\vartheta}\right)\left(g^{\prime}\right) & =\omega\left(g g^{\prime}\right) T_{g}=P_{g g^{\prime}} \Omega\left(g g^{\prime}\right) T_{。} \\
& =P_{o g^{\prime}}\left(\Omega T_{g}\right) g^{\prime}=P_{g} P_{g^{\prime}} \Omega\left(g^{\prime}\right)=P_{\theta} \omega\left(g^{\prime}\right) \\
& =\left(P_{0} \omega\right)\left(g^{\prime}\right) .
\end{aligned}
$$

Hence $\omega T_{o}=P_{o} \omega$ and $\omega$ is equivariant. This completes the proof of the first 
part of the theorem and incidentally shows that every equivariant $V$-form on $G$ is analytic.

We proceed with the proof of (10.1). Differentiating (10.2) we have

$$
d \omega=\sum_{i, j}\left(a_{i j} d \Omega_{j}+d a_{i j} \square \Omega_{j}\right) v_{i} .
$$

Hence

$$
\begin{aligned}
(d \omega)_{e} & =\sum_{i, j}\left(a_{i j} d \Omega_{j}\right)_{e} v_{i}+\sum_{i, j}\left(d a_{i j}\right)_{e} \square\left(\Omega_{j}\right)_{e} v_{i} \\
& =(d \Omega)_{e}+\sum_{i, j}\left(d a_{i j}\right)_{e} \square\left(\Omega_{j}\right)_{e} v_{i} .
\end{aligned}
$$

Applying both sides to $x_{1}, \cdots, x_{q+1}$ in the Lie algebra $L$ we have

$$
\begin{aligned}
\{d \omega\}\left(x_{1}, \cdots, x_{q+1}\right)= & \{d \Omega\}\left(x_{1}, \cdots, x_{q+1}\right) \\
& +\sum_{i, j}\left\{d a_{i j}\right\} \square\left\{\Omega_{j}\right\}\left(x_{1}, \cdots, x_{q+1}\right) v_{i} .
\end{aligned}
$$

The first term on the right-hand side is, by Theorem 9.1,

$$
\begin{aligned}
\{d \Omega\}\left(x_{1}, \cdots, x_{q+1}\right) & \\
\quad & \frac{1}{q+1} \sum_{k<l}(-1)^{k+l+1}\{\Omega\}\left(\left[x_{k}, x_{l}\right], x_{1}, \cdots, \hat{x}_{k}, \cdots, \hat{x}_{l}, \cdots, x_{q+1}\right) \\
& =\frac{1}{q+1} \sum_{k<l}(-1)^{k+l+1}\{\omega\}\left(\left[x_{k}, x_{l}\right], x_{1}, \cdots, \hat{x}_{k}, \cdots, \hat{x}_{l}, \cdots, x_{q+1}\right) .
\end{aligned}
$$

The second term evaluates as follows

$$
\begin{aligned}
\sum_{i, j}\left\{d a_{i j}\right\} \square\left\{\Omega_{j}\right\}\left(x_{1}, \cdots, x_{q+1}\right) v_{i} \\
=A\left[\sum_{i, j}\left\{d a_{i j}\right\}\left(x_{1}\right)\left\{\Omega_{j}\right\}\left(x_{2}, \cdots, x_{q+1}\right) v_{i}\right] \\
=A\left[F\left(x_{1}\right)\{\Omega\}\left(x_{2}, \cdots, x_{q+1}\right)\right] \\
=A\left[P\left(x_{1}\right)\{\omega\}\left(x_{2}, \cdots, x_{q+1}\right)\right] \\
=\frac{1}{q+1} \sum_{k}(-1)^{k+1} P\left(x_{k}\right)\{\omega\}\left(x_{1}, \cdots, \hat{x}_{k}, \cdots, x_{q+1}\right) .
\end{aligned}
$$

This completes the proof of Theorem 10.1 .

11. Invariance under right translations. Given an element $h \in G$ we consider the right translation $R_{h}: G \rightarrow G$ defined by $R_{h} g=g h$. Let $P$ be a representation of $G$ with representation space $V$ and let $\omega$ be an equivariant $V$-form of order $q$ on $G$. Consider the $V$-form $\omega R_{h}$. We have

$$
\omega R_{h} T_{\diamond}=\omega T_{0} R_{h}=P_{\ominus}\left(\omega R_{h}\right)
$$


which shows that $\omega R_{h}$ is equivariant. If we further denote by $C_{h}$ the inner automorphism $g \rightarrow h g h^{-1}$ induced by $h$ then

$$
\omega R_{h}=P_{h} \omega T_{h^{-1}} R_{h}=P_{h} \omega C_{h^{-1}} .
$$

This proves that $\omega=\omega R_{h}$ if and only if $\omega C_{h}=P_{h} \omega$. Since both $\omega$ and $\omega R_{h}$ are equivariant, the relation $\omega=\omega R_{h}$ is equivalent with $\omega_{e}=\left(\omega R_{h}\right)_{e}$ which in turn is equivalent with $\left(\omega C_{h}\right)_{e}=\left(P_{h} \omega\right)_{e}$. Hence we see that a necessary and suffcient condition for $\omega=\omega R_{h}$ is that

$$
\{\omega\}\left(C_{h} x_{1}, \cdots, C_{h} x_{q}\right)=P_{h}\{\omega\}\left(x_{1}, \cdots, x_{q}\right)
$$

where $x_{1}, \cdots, x_{q} \in L$ and $C_{h}$ is the automorphism of $L$ induced by the inner automorphism $C_{h}$.

THEOREM 11.1. Let $H$ be a closed and connected subgroup of $G$, and let $L_{H}$ be the corresponding subalgebra of $L$. Let $\omega$ be an equivariant $V$-form of order $q$ on $G$ relative to a representation $P$. Then $\omega=\omega R_{h}$ for all $h \in H$ if and only if

$$
\begin{aligned}
& P\left(x^{\prime}\right)\{\omega\}\left(x_{1}, \cdots, x_{q}\right) \\
& \quad=\{\omega\}\left(\left[x_{1}, x^{\prime}\right], x_{2}, \cdots, x_{q}\right)+\cdots+\{\omega\}\left(x_{1}, \cdots, x_{q-1},\left[x_{q}, x^{\prime}\right]\right)
\end{aligned}
$$

for all $x^{\prime} \in L_{H}$ and $x_{1}, \cdots, x_{q} \in L$.

Proof. Let $\mathfrak{A}^{a}$ be the vector space of all $q$-linear alternating functions on $L$ with values in $V$. Given $h \in H$ and $f \in \mathscr{A}^{a}$, define the elements $P_{h} f$ and $Q_{h} f$ of $\mathfrak{A}^{a}$ by setting

$$
\begin{aligned}
& \left(P_{h} f\right)\left(x_{1}, \cdots, x_{q}\right)=P_{h}\left(f\left(x_{1}, \cdots, x_{q}\right)\right), \\
& \left(Q_{h} f\right)\left(x_{1}, \cdots, x_{q}\right)=f\left(C_{h} x_{1}, \cdots, C_{h} x_{q}\right) .
\end{aligned}
$$

Both correspondences $P_{h}: f \rightarrow P_{h} f$ and $Q_{h}: f \rightarrow Q_{h} f$ give linear representations $P$ and $Q$ of $H$ with $\mathscr{A}^{q}$ as representation space. We are interested in characterizing those $f \in \mathfrak{A}^{a}$ for which

$$
Q_{h} f=P_{h} f \quad \text { for all } h \in H .
$$

Let $h=\exp \left(t x^{\prime}\right)$ for $x^{\prime} \in L_{H}$. Consider

$$
Q_{x^{\prime}} f=\lim _{t \rightarrow 0} \frac{Q_{h} f-f}{t}, \quad P_{x^{\prime}} f=\lim _{t \rightarrow 0} \frac{P_{h} f-f}{t} .
$$

Clearly (11.3) implies

$$
Q_{x^{\prime} f}=P_{x^{\prime}} f \quad \text { for all } x^{\prime} \in L_{H} .
$$

Conversely (11.4) implies (11.3) for all $h$ in a neighbourhood of $e$ in $H$ [LG, p. 118] and since $H$ is connected this implies (11.3) for all $h \in H$. We have

$$
\left(P_{x^{\prime} f}\right)\left(x_{1}, \cdots, x_{q}\right)=P_{x^{\prime}}\left(f\left(x_{1}, \cdots, x_{q}\right)\right) .
$$


Since [LG, p. 124]

$$
\left[x, x^{\prime}\right]=\lim _{t \rightarrow 0} \frac{C_{h} x-x}{t}
$$

and

$$
\left(Q_{x^{\prime}} f\right)\left(x_{1}, \cdots, x_{q}\right)=\lim _{t \rightarrow 0} \frac{f\left(C_{h} x_{1}, \cdots, C_{h} x_{q}\right)-f\left(x_{1}, \cdots, x_{q}\right)}{t}
$$

it follows from the linearity of $f$ that

$$
\begin{aligned}
\left(Q_{x^{\prime}} f\right)\left(x_{1},\right. & \left.\cdots, x_{q}\right) \\
& =f\left(\left[x_{1}, x^{\prime}\right], x_{2}, \cdots, x_{q}\right)+\cdots+f\left(x_{1}, \cdots, x_{q-1},\left[x_{q}, x^{\prime}\right]\right) .
\end{aligned}
$$

Propositions (11.4)-(11.6) combine to give formula (11.2).

12. Invariant forms. A differential form $\omega$ on a Lie group $G$ will be called invariant if it is both left and right invariant. It follows from Theorem 11.1 that

(12.1) $A$ left invariant form $\omega$ of order $q$ on a connected Lie group $G$ is invariant if and only if

$$
\{\omega\}\left(\left[x_{1}, x\right], x_{2}, \cdots, x_{q}\right)+\cdots+\{\omega\}\left(x_{1}, \cdots, x_{q-1},\left[x_{q}, x\right]\right)=0
$$

for all $x, x_{1}, \cdots, x_{q} \in L$.

Assuming that $\omega$ is invariant we compute $\{d \omega\}$.

$$
\begin{aligned}
\{d \omega\}\left(x_{1}, \cdots, x_{q+1}\right) & \\
& =\frac{1}{q+1} \sum_{i<j}(-1)^{i+j+1}\{\omega\}\left(\left[x_{i}, x_{j}\right], x_{1}, \cdots, \hat{x}_{i}, \cdots, \hat{x}_{j}, \cdots, x_{q+1}\right) \\
& =\frac{1}{2(q+1)} \sum_{i \neq j} \epsilon_{i j}\{\omega\}\left(\left[x_{i}, x_{i}\right], x_{1}, \cdots, \hat{x}_{i}, \cdots, \hat{x}_{i}, \cdots, x_{q+1}\right)
\end{aligned}
$$

where $\epsilon_{i j}=(-1)^{i+j+1}$ if $i<j$, and $\epsilon_{i j}=(-1)^{i+j}$ if $i>j$. For each fixed $j=1, \cdots$, $q+1$ the sum vanishes in virtue of (12.2). Hence $\{d \omega\}=0$ and

(12.3) Every invariant form is closed.

Assume now that the Lie group $G$ is compact and connected. Theorem 7.1 implies that the cohomology ring of $G$ obtained using invariant forms only is isomorphic (under the natural inclusion homomorphism) with the cohomology ring of $G$ obtained using all the regular differential forms. Combining this with (12.3) we find:

THEOREM 12.1. Let $G$ be a compact and connected Lie group. Every cohomology class of differential forms on $G$ contains precisely one invariant form. The invariant forms span a ring isomorphic with the conomology ring of the manifold $G$. 
If in addition to being compact and connected $G$ is also semi-simple then $G$ possesses a Riemannian metric invariant with respect to both left and right translations. Moreover Hodge $\left({ }^{13}\right)$ has shown that the harmonic forms with respect to this Riemannian metric coincide with the forms that are invariant. Thus Theorem 12.1 gives a relatively simple proof of Hodge's theorems on harmonic forms on a Riemannian manifold in the case where this manifold is the group manifold of a semi-simple compact group.

13. The case of a homogeneous space. We now return to the consideration of a homogenous space $G / H=M, H$ being a closed subgroup of the Lie group $G$. We denote by $\Pi$ the mapping $g \rightarrow g H$ of $G$ onto $M$. We use the symbols $T_{\theta}$ and $R_{\theta}$ to denote the left and right translations of $G$ while $\widetilde{T}_{\sigma}$ will denote the transformations $g^{\prime} H \rightarrow g g^{\prime} H$ of $M$ onto itself. We have

$$
\begin{aligned}
& \Pi T_{g}=\tilde{T}_{g} \Pi \\
& \Pi R_{h}=\Pi
\end{aligned}
$$

for $h \in H$.

Given any $V$-form $\omega$ on $M$ we consider the $V$-form $\omega \Pi$ on $G$. We have

$$
\begin{aligned}
d(\omega \Pi) & =(d \omega) \Pi, \\
\omega \Pi & =0 \quad \text { if and only if } \omega=0 .
\end{aligned}
$$

Let $P$ be a representation of $G$ with $V$ as representation space.

(13.5) $\omega \Pi$ is equivariant if and only if $\omega$ is.

In fact, $P_{o}(\omega \Pi)=\left(P_{0} \omega\right) \Pi$ and $(\omega \Pi) T_{o}=\left(\omega \tilde{T}_{o}\right) \Pi$. Hence (13.4) implies that $P_{g}(\omega \Pi)=(\omega \Pi) T_{0}$ if and only if $P_{0} \omega=\omega \widetilde{T}_{0}$.

We shall now characterize the $V$-forms on $G$ of the form $\omega \Pi$.

Let $g \in G$. Consider the tangent vector spaces $V(g)$ at $g$ and $V(\Pi g)$ at $\Pi g$. The linear mapping $d \Pi: V(g) \rightarrow V(\Pi g)$ is then a mapping onto.

(13.6) Given a $V$-form $\Omega$ of order $q$ on $G$ there is $a V$-form $\omega$ on $M$ such that $\Omega=\omega \Pi$ if and only if the following two conditions hold:

(13.7) $\Omega_{g}\left(x_{1}, \ldots, x_{q}\right)=0$ if $d \Pi x_{i}=0$ for at least one of the vectors $x_{i} \in V(g)$.

(13.8) $\Omega R_{h}=\Omega$ for all $h \in H$.

The necessity of (13.7) is obvious. If $\Omega=\omega \Pi$ then $\Omega R_{h}=\omega \Pi R_{h}=\omega \Pi=\Omega$ which proves (13.8).

Assume now that $\Omega$ is given satisfying (13.7) and (13.8). Given a point $m \in M$ select $g \in G$ so that $\Pi g=m$. Given vectors $x_{1}, \cdots, x_{q} \in V(m)$ select vectors $\bar{x}_{1}, \cdots, \bar{x}_{q} \in V(g)$ such that $d \Pi \bar{x}_{i}=x_{i}$. Define $\omega_{m}\left(x_{1}, \cdots, x_{q}\right)$ $=\Omega_{g}\left(\bar{x}_{1}, \cdots, \bar{x}_{q}\right)$. It follows from the linearity of $\Omega_{g}$ and from (13.7) that $\omega_{m}\left(x_{1}, \cdots, x_{q}\right)$ does not depend upon the choice of $\bar{x}_{1}, \cdots, \bar{x}_{q}$. If we replace $g$ by $g h$ for some $h \in H$, then (13.7) implies that

$$
\Omega_{g}\left(\bar{x}_{1}, \cdots, \bar{x}_{q}\right)=\left(\Omega R_{h}\right)_{g}\left(\bar{x}_{1}, \cdots, \bar{x}_{q}\right)=\Omega_{g h}\left(d R_{h} \bar{x}_{1}, \cdots, d R_{h} \bar{x}_{q}\right) .
$$

(13) W. V. D. Hodge, The theory and applications of harmonic integrals, Cambridge University Press, 1941. 
Since $d \Pi d R_{h} \bar{x}_{i}=x_{i}$, this proves that $\omega_{m}\left(x_{1}, \cdots, x_{q}\right)$ is independent of the choice of $g$. It is clear that $\omega$ is a $V$-form such that $\Omega=\omega \Pi$. Moreover $\omega$ is analytic if $\Omega$ is.

Theorem 13.1. Let $G$ be a connected Lie group and $H$ a closed connected subgroup of $G$. Denote by $L$ and $L_{H}$ the Lie algebras of $G$ and $H$ respectively, by $M$ the homogenous space $G / H$, and by $\Pi$ the mapping $g \rightarrow g H$ of $G$ onto $M$. Consider $G$ as a group of operators for $M$ by setting $\widetilde{T}_{o}\left(g^{\prime} H\right)=g g^{\prime} H$. Let $P$ be a representation of $G$ and $V$ the representation space of $V$.

The mapping $\omega \rightarrow\{\omega \Pi\}$ establishes a 1-1 correspondence between the equivariant $V$-forms $\omega$ of order $q$ on $M$ and those q-linear alternating functions $\{\Omega\}$ on $L$ which satisfy the conditions

$$
\begin{aligned}
\{\Omega\}\left(x_{1}, \cdots, x_{q}\right)= & 0 \text { if at least one } x_{i} \text { is in } L_{H}, \\
P(x)\{\Omega\}\left(x_{1}, \cdots, x_{q}\right)= & \{\Omega\}\left(\left[x_{1}, x\right], x_{2}, \cdots, x_{q}\right)+\cdots \\
& +\{\Omega\}\left(x_{1}, \cdots, x_{q-1}\left[x_{q}, x\right]\right)
\end{aligned}
$$

for $x \in L_{H}, x_{1}, \cdots, x_{q} \in L$.

To prove Theorem 13.1 we only need to remark that (13.9) and (13.10) are equivalent with (13.7) and (13.8). In fact, if $\Omega$ is equivariant, condition (13.7) need only be stated for $g=e$ and then it becomes condition (13.9) since $L_{H}$ is the kernel of the linear mapping $L=V \cdot(e) \rightarrow V(\Pi e)$. Conditions (13.8) and (13.10) are equivalent in view of Theorem 11.1.

\section{Chapter III. Cohomology groups of Lie algebras}

14. The cohomology ring of a Lie algebra. Let $L$ be a Lie algebra over a field $K$ of characteristic 0 . We denote by $C(L)$ the contravariant Grassmann algebra over $L$, and by $C^{q}(L)$ the space of elements of order $q$ of $C(L)$. The elements of $C^{q}(L)$ for $q>0$ are the $q$-linear alternating functions in $L$ with values in $K$ and $C^{0}(L)=K$. The elements of $C^{g}(L)$ will also be called $q$-dimensional cochains in $L$. For two cochains $f_{1} \in C^{p}(L), f_{2} \in C^{q}(L)$ the product $f_{1} \square f_{2} \in C^{p+q}(L)$ is the Grassmann or alternating product

$$
\left(f_{1} \square f_{2}\right)\left(x_{1}, \cdots, x_{p+q}\right)=A\left(f_{1}\left(x_{1}, \cdots, x_{p}\right) f_{2}\left(x_{p+1}, \cdots, x_{p+q}\right)\right)
$$

where $A$ stands for the alternating operator applied to the variables $x_{1}, \cdots, x_{p+q} \in L$.

Guided by the formula (9.2), we define for each cochain $f \in C^{a}(L)$ a cochain $\delta f \in C^{q+1}(L)$ called the coboundary of $f$ as follows

$$
\begin{aligned}
& (\delta f)\left(x_{1}, \cdots, x_{q+1}\right) \\
& \quad=\frac{1}{q+1} \sum_{k<l}(-1)^{k+l+1} f\left(\left[x_{k}, x_{l}\right], x_{1}, \cdots, \hat{x}_{k}, \cdots, \hat{x}_{l}, \cdots, x_{q+1}\right) .
\end{aligned}
$$

If $q=0$ we set $\delta f=0$. As we saw in $\S 9, \delta f$ can be defined alternatively as 


$$
\delta f\left(x_{1}, \cdots, x_{q+1}\right)=\frac{q}{2} A\left(f\left(\left[x_{1}, x_{2}\right], x_{3}, \cdots, x_{q+1}\right)\right) .
$$

The operation $\delta$ maps $C^{q}(L)$ linearly into $C^{q+1}(L)$. We shall show that for $f_{1} \in C^{p}(L), f_{2} \in C^{q}(L)$

$$
\delta\left(f_{1} \square f_{2}\right)=\left(\delta f_{1}\right) \square f_{2}+(-1)^{p} f_{1} \square \delta f_{2} .
$$

In the case $p=0$, the formula is obvious. The case $p=1$ follows from exactly the same computation as the one made in $\S 9$. From now on we proceed by induction. Assume that (14.2) is valid for $f_{1}^{\prime} \in C^{p-1}(L)$. Let $f \in C^{1}(L)$ and $f_{1}=f \square f_{1}^{\prime}$. Then

$$
\begin{aligned}
\delta\left(f_{1} \square f_{2}\right) & =\delta\left(f \square f_{1}^{\prime} \square f_{2}\right)=(\delta f) \square f_{1}^{\prime} \square f_{2}-f \square \delta\left(f_{1}^{\prime} \square f_{2}\right) \\
& =(\delta f) \square f_{1}^{\prime} \square f_{2}-f \square\left(\delta f_{1}^{\prime}\right) \square f_{2}+(-1)^{p} f \square f_{2}^{\prime} \square \delta f_{2} \\
& =\delta\left(f \square f_{1}^{\prime}\right) \square f_{2}+(-1)^{p}\left(f \square f_{1}^{\prime}\right) \square \delta f_{2} \\
& =\left(\delta f_{1}\right) \square f_{2}+(-1)^{p} f_{1} \square \delta f_{2} .
\end{aligned}
$$

This proves (14.2) for any $f_{1}$ of the form $f \square f_{1}^{\prime}$. It follows by linearity that (14.2) holds for all $f_{1} \in C^{p}(L)$.

We next prove for $f \in C^{q}(L)$

$$
\delta \delta f=0 .
$$

The formula is obvious for $q=0$. For $q=1$ we have

$$
\begin{aligned}
6(\delta \delta f)\left(x_{1}, x_{2}, x_{3}\right) & =2 \delta f\left(\left[x_{1}, x_{2}\right], x_{3}\right)+2 \delta f\left(\left[x_{2}, x_{3}\right], x_{1}\right)-2 \delta f\left(\left[x_{1}, x_{3}\right], x_{2}\right) \\
& =f\left(\left[\left[x_{1}, x_{2}\right], x_{3}\right]+\left[\left[x_{2}, x_{3}\right], x_{1}\right]+\left[\left[x_{3}, x_{1}\right], x_{2}\right]\right)=0 .
\end{aligned}
$$

For $q>1$ we proceed by induction. Assume first that $f=f_{1} \square f_{2}$ where $f_{1} \in C^{1}(L)$, $f_{2} \in C^{p-1}(L)$. Then by (14.2) $\delta f=\left(\delta f_{1}\right) \square f_{2}-f_{1} \square \delta f_{2}$ and $\delta \delta f=\left(\delta \delta f_{1}\right) \square f_{2}+\left(\delta f_{1}\right)$ $\square \delta f_{2}-\left(\delta f_{1}\right) \square \delta f_{2}+f_{1} \square \delta \delta f_{2}=0$. It follows by linearity that (14.3) holds for every $f \in C^{q}(L)$.

Having established (14.3) we proceed with the familiar definitions of combinatorial topology. A cochain $f$ is a cocycle provided $\delta f=0$. The cocycles of dimension $q$ form a subspace $Z^{q}(L)$ of $C^{q}(L)$. A cochain $f \in C^{q}(L)$ is a coboundary if it is of the form $\delta f^{\prime}$ for some $f^{\prime} \in C^{q-1}(L)$. The coboundaries form a subspace $B^{q}(L)$ of $Z^{q}(L)$. If $q=0$ then $B^{q}(L)=0$ by definition. The factor space $H^{q}(L)=Z^{q}(L) / B^{q}(L)$ is called the qth cohomology group of the Lie algebra $L$.

If $f_{1} \in Z^{p}(L), f_{2} \in Z^{q}(L)$ it follows from (14.2) that $f_{1} \square f_{2} \in Z^{p+q}(L)$; if furthermore either $f_{1} \square B^{p}(L)$ or $f_{2} \square B^{q}(L)$ then $f_{1} \square f_{2} \in B^{p+q}(L)$. It follows that a multiplication between elements of $H^{p}(L)$ and $H^{q}(L)$ with values in $H^{p+q}(L)$ is defined and that, $n$ being the dimension of $L$, the direct sum $\sum_{p=0}^{n} H^{p}(L)$ is thus made into a ring $H(L)$ called the cohomology ring of the Lie algebra $L$.

For $q=0$ we have $Z^{0}=C^{0}=K$ and $B^{0}=0$ so that 


$$
H^{0}(L)=K \text {. }
$$

For $q=1$ we have $B^{1}=0$ so that $H^{1}=Z^{1}$. If $f \in C^{1}(L)$ then $(\delta f)\left(x_{1}, x_{2}\right)$ $=2^{-1} f\left(\left[x_{1}, x_{2}\right]\right)$. If we therefore denote by $[L, L]$ the subalgebra of $L$ spanned by elements of the form $\left[x_{1}, x_{2}\right]$, then it appears that $f$ is a cocycle if and only if it vanishes on $[L, L]$. Hence

(14.5) $H^{1}(L)$ is the conjugate space of $L /[L, L]$.

We observe that if $n$ is the dimension of $L$ and $q>n$ then every $q$-dimensional cochain is identically zero and $H^{q}(L)=0$.

It is worth observing that an identical cohomology ring is obtained by dropping the factor $1 /(q+1)$ in (14.1). In this modified form the preceding definitions apply to Lie algebras over a field of any characteristic.

II 15. Connections with Lie groups. Theorem 9.1 implies the following theorem.

THEOREM 15.1. If $L$ is the Lie algebra of the Lie group $G$, then $H^{q}(L)$ is isomorphic with the cohomology group $E^{q}(G)$ obtained using the left invariant differential forms on $G$. The ring $H(L)$ is isomorphic with the ring $E(G)$.

Applying Theorem 2.3 and de Rham's theorem we find:

THEOREM 15.2. If $L$ is the Lie algebra of the compact and connected Lie group $G$, then $H^{q}(L)$ is isomorphic with the qth cohomology group $H^{q}(G)$ with real coefficients and the ring $H(L)$. is isomorphic with the cohomology ring $H(G)$ of $G$.

As a corollary we obtain the following generalization of a theorem of Pontrjagin (14).

THEOREM 15.3. Two locally isomorphic compact connected Lie groups have isomorphic cohomology rings.

16. Semi-simple Lie algebras. Let $L$ be a Lie algebra over a field $K$ of characteristic 0 . A representation $P$ of $L$ with a vector space $V$ as representation space will be called fully reducible if to every $P$-invariant subspace $V_{1}$ of $V$ there is a $P$-invariant subspace $V_{2}$ of $V$ such that $V$ is the direct sum $V_{1}+V_{2}$. If every representation of $L$ is fully reducible then $L$ is called semisimple.

In particular consider the adjoint representation defined by ad $(x): y$ $\rightarrow[y, x]$ for which the linear space of $L$ is the representation space. The invariant subspaces are then precisely the ideals of $L$. Hence

(16.1) If $L$ is semi-simple then for every ideal $L_{1}$ in $L$ there is an ideal $L_{2}$ in $L$ such that $L$ is the direct sum $L_{1}+L_{2}$.

Using (16.1) we prove

(14) L. Pontrjagin, Homologies in compact Lie groups, Rec. Math. (Mat. Sbornik) N.S. vol. 6 (1939) pp. $389-422$. 
(16.2) If $L$ is semi-simple and $L_{1}$ is an ideal in $L$ then both $L_{1}$ and $L / L_{1}$ are semi-simple.

Proof. Using the natural homomorphism $L \rightarrow L / L_{1}$ every representation of $L / L_{1}$ gives a representation of $L$. Since the representation of $L$ thus obtained is fully reducible the same holds for the representation of $L / L_{1}$. To prove that $L_{1}$ is semi-simple we observe that, in virtue of (16.1), $L_{1}$ is isomorphic with $L / L_{2}$ where $L_{2}$ is an ideal of $L$.

(16.3) A semi-simple Lie algebra $L$ has center 0.

Assume that $L$ is semi-simple and has a nonzero center. Since the center is an ideal, we may assume in virtue of (16.2) that $L$ is its own center. Let $x_{1}, \cdots, x_{n}$ be a base in $L$ and let $V$ be a 2 -dimensional vector space with generators $v_{1}, v_{2}$. Define a representation $P$ of $L$ in $V$ by setting

$$
P_{x_{1} v_{1}}=v_{2}, \quad P_{x_{1} v_{2}}=0, \quad P_{x_{i}} v_{j}=0 \quad \text { for } i=2, \cdots, n ; j=1,2 .
$$

In this representation the subspace $V_{2}$ generated by $v_{2}$ is invariant, but since $P_{x_{1}}\left(\alpha v_{1}+\beta v_{2}\right)=\alpha v_{2}$ for $\alpha, \beta \in K$, no other 1-dimensional subspace is invariant. Hence $L$ is not semi-simple.

An alternative way of formulating (16.3) is

(16.4) The adjoint representation of a semi-simple Lie algebra is faithful.

(16.5) If $L$ is semi-simple then $L=[L, L]$.

Indeed, $[L, L]$ is an ideal. Therefore, by (16.2), $L /[L, L]$ is semi-simple. Hence, by (16.3), $L /[L, L]=\{0\}$.

From (16.5) and (14.5) we deduce

(16.6) If $L$ is semi-simple then $H^{1}(L)=\{0\}$.

Using (16.6) we shall now prove the following theorem.

THEOREM 16.1. A compact connected Lie group $G$ is semi-simple if and only if its fundamental group is finite $\left({ }^{16}\right)$.

Proof. Let $L$ be the Lie algebra of $G$. Assume that $G$ is semi-simple. Then $L$ is semi-simple and, by $(16.6), H^{1}(L)=\{0\}$. Hence, by Theorem 15.2, $H^{1}(G)=\{0\}$ and the first Betti number of $G$ is zero. This implies that the 1-dimensional homology group of $G$ with integral coefficients is finite. However, this group is isomorphic with the fundamental group of $G$, since this fundamental group is abelian.

Conversely assume that the fundamental group of $G$ is finite. Then the universal covering group $\tilde{G}$ of $G$ is compact. Since $\bar{G}$ is simply connected, every representation of the Lie algebra $L$ is induced by some representation of $\tilde{G}$ [LG, p. 113]. $\tilde{G}$ being compact, every representation of $\tilde{G}$ is fully reducible. Hence the same follows for every representation of $L$. Hence $L$ is semi-simple and so is $G$.

(15) Cf. H. Samelson, A note on Lie groups, Bull. Amer. Math. Soc. vol. 52 (1946) pp. 870873. 
17. The unitary trick. A semi-simple Lie algebra (over the field of reals) will be called compact if it is the Lie algebra of some compact connected Lie group. Any proposition concerning the cohomology rings of compact connected semi-simple Lie groups translates in virtue of Theorem 15.2 into a similar proposition concerning the cohomology rings of compact Lie algebras. The "unitary trick" is a general method which then allows us to extend the proposition to arbitrary semi-simple Lie algebras over any field of characteristic 0 .

Let $L$ be a Lie algebra over a field $K$ (of characteristic 0 ) and let $N$ be an extension of $K$. We shall denote by $L_{N}$ the Lie algebra obtained from $L$ by extending the ground field from $K$ to $N$. $L$ is semi-simple if and only if $L_{N}$ is semi-simple.

A property $P$ of Lie algebras will be called linear provided: $1^{\circ}$ if a Lie algebra $L$ has the property $P$, then so does $L_{N}$ for any extension $N$ of the ground field; $2^{\circ}$ if $L_{N}$ has the property $P$ for some extension $N$ of the ground field then $L$ has property $P$.

The essence of the unitary trick is then embodied in the following theorem.

TheOREM 17.1. Let $P$ be a linear property. If all compact Lie algebras have property $P$ then all semi-simple Lie algebras have property $P$.

Proof. First assume that $L$ is a semi-simple Lie algebra over the field $C$ of complex numbers. $\mathrm{H}$. Weyl has proved $\left({ }^{16}\right)$ that there is a compact Lie algebra $L^{\prime}$ such that $L_{c}^{\prime}$ is isomorphic with $L$. Hence $L$ has the property $P$.

Next assume that $L$ is a semi semi-simple Lie algebra over a field $K$ which can be deduced from the field of rationals by the adjunction of a finite number of elements. Then $K$ may be regarded as a subfield of $C$. Since $L_{C}$ is semisimple it has the property $P$ and therefore $L$ has the property $P$.

Finally let $L$ be a semi-simple Lie algebra over an arbitrary field $K$ of characteristic 0 , and let $x_{1}, \cdots, x_{n}$ be a base of $L$. We then have

$$
\left[x_{i}, x_{j}\right]=\sum_{k=1}^{n} c_{i j k} x_{k}, \quad c_{i j k} \in K .
$$

Let $K_{0}$ be the smallest subfield of $K$.containing the quantities $c_{i j k}$. Then the vector space $L_{0}=K_{0} x_{1}+K_{0} x_{2}+\cdots+K_{0} x_{n}$ has the structure of a Lie algebra over $K_{0}$, the brackets $\left[x_{i}, x_{j}\right]$ having the same meaning in $L_{0}$ as in $L$. It is easily seen that $L_{0}$ is semi-simple and that $\left(L_{0}\right)_{K}=L$. Since the field $K_{0}$ can be deduced from the field of rationals by the adjunction of a finite number of elements, it follows that $L_{0}$ has the property $P$ and therefore $L$ also has the property $P$.

18. Hopf's theorem. We shall apply the unitary trick to carry over cer-

(16) H. Weyl, Theorie der Darstellung kontinuierlichen half-einfacher Gruppen durch lineare Transformationen II, Math. Zeit. vol. 24 (1926) pp. 328-376; Satz 6, p. 375. 
tain theorems proved by $\mathrm{H}$. Hopf $\left({ }^{17}\right)$ for compact Lie groups to semi-simple Lie algebras. Hopf's theorem asserts that the cohomology ring $H(G)$ of a compact connected Lie group is isomorphic with the direct sum of cohomology rings of a finite number of odd-dimensional spheres.

THEOREM 18.1. The cohomology ring $H(L)$ of a semi-simple Lie algebra $L$ over a field $K$ of characteristic 0 is isomorphic with the direct sum of the cohomology rings (over $K$ ) of a finite number of odd-dimensional spheres.

Proof. Hopf's theorem combined with Theorem 15.2 gives a proof for compact Lie algebras. It therefore remains to verify that the property described in the conclusion of the theorem is linear. Let then $N$ be an extension of the ground field $K$. A simple argument shows then that the cohomology ring $H\left(L_{N}\right)$ regarded as an algebra over $N$ is obtained from $H(L)$ by extending the groundfield from $K$ to $N$. Hence if the conclusion of the theorem holds for $H(L)$ it also holds for $H\left(L_{N}\right)$ and vice versa.

Let $p_{q}$ denote the $q$ th Betti number of $L$, that is, the dimension over $K$, of the vector space $H^{q}(L)$. The polynomial

$$
P_{L}(t)=p_{0}+p_{1} t+p_{2} t^{2}+\cdots+p_{n} t^{n},
$$

where $n$ is the dimension of $L$, is then called the Poincaré polynomial of $L$. Theorem 18.1 implies that if $L$ is semi-simple then

$$
P_{L}(t)=\left(1+t^{m_{1}}\right) \cdots\left(1+t^{m_{l}}\right)
$$

where $m_{1}, \cdots, m_{l}$ are odd integers. Substituting $t=-1$ in (18.1) and (18.2) shows that

$$
\sum_{q=0}^{n}(-1)^{q} p_{q}=0
$$

which is the algebraic counterpart of the known fact that the Euler characteristic of a compact Lie group is 0 . Substituting $t=1$ we find

$$
\sum_{q=0}^{n} p_{q}=2^{l} \text {. }
$$

The quantity $l$ in the case of a compact Lie group is called the rank of $G$ and is the maximal dimension of the abelian subgroups of $G$.

Since we already know that $H^{1}(L)=\{0\}$ for $L$ semi-simple it follows that $m_{i} \geqq 3$ for $i=1, \cdots, l$. Consequently

(18.3) If $L$ is semi-simple then $H^{q}(L)=\{0\}$ for $q=1,2,4$.

The case $q=2$ will be given an algebraic proof later. We shall also show that $H^{3}(L) \neq\{0\}$.

(17) H. Hopf, Über die Topologie der Gruppen-Mannigfaltigkeiten und ihre Verallgemeinerungen, Ann. of Math. vol. 42 (1941) pp. 22-52. 
19. Invariant cochains. Guided by the results of (12.1) we define a cochain $f \in C^{q}(L)$ to be invariant if

$$
f\left(\left[x_{1}, x\right], x_{2}, \cdots, x_{q}\right)+\cdots+f\left(x_{1}, \cdots, x_{q-1},\left[x_{q}, x\right]\right)=0
$$

for all $x, x_{1}, \cdots, x_{q} \in L$. The same computation as in $\$ 12$ shows that

(19.2) Every invariant cochain is a cocycle.

THEOREM 19.1. Let $L$ be a semi-simple Lie algebra over a field of characteristic 0 . Every cohomology class of $H^{q}(L)$ contains exactly one invariant cocycle. The invariant cocycles constitute a ring isomorphic with the cohomology ring $H(L)$.

This theorem could be derived from Theorem 12.1 by the application of the unitary trick. The following proof is purely algebraic.

In the following lemmas the semi-simplicity of $L$ is not assumed.

For each $x \in L$ we define a linear mapping $d_{x}: C^{q}(L) \rightarrow C^{q}(L)$ as follows

$$
\begin{aligned}
\left(d_{x} f\right)\left(x_{1}, \cdots, x_{q}\right) & \\
& =f\left(\left[x_{1}, x\right], x_{2}, \cdots, x_{q}\right)+\cdots+f\left(x_{1}, \cdots, x_{q-1},\left[x_{q}, x\right]\right)
\end{aligned}
$$

and $d_{x} f=f$ if $q=0$.

Condition (19.1) can now be written as

$$
d_{x} f=0 .
$$

We shall establish the following properties of $d_{x}$ :

$$
\begin{aligned}
d_{x}\left(f_{1} \square f_{2}\right) & =\left(d_{x} f_{1}\right) \square f_{2}+f_{1} \square d_{x} f_{2} ; \\
d_{y} d_{x} f-d_{x} d_{y} f & =d_{[x, y]} f ; \\
d_{x}(\delta f) & =\delta\left(d_{x} f\right) ;
\end{aligned}
$$

(19.7) If $f$ is a cocycle then $d_{x} f$ is a coboundary.

$\operatorname{Ad}(19.4)$. The formula is obvious if either $f_{1}$ or $f_{2}$ has degree zero. Let then $f_{1} \in C^{1}(L)$ and $f_{2} \in C^{q}(L), q>0$. From the definition of the Grassmann multiplication

$$
\left(f_{1} \square f_{2}\right)\left(x_{1}, \cdots, x_{q+1}\right)=\frac{1}{q+1} \sum_{i=1}^{q+1}(-1)^{i+1} f_{1}\left(x_{i}\right) f_{2}\left(x_{1}, \cdots, \hat{x}_{i}, \cdots, x_{q+1}\right)
$$

hence

$$
\begin{aligned}
{\left[d_{x}\left(f_{1} \square f_{2}\right)\right]\left(x_{1}, \cdots,\right.} & \left.x_{q+1}\right) \\
= & -\frac{1}{q+1} \sum_{i=1}^{q+1}(-1)^{i+1} f_{1}\left(\left[x_{i}, x\right]\right) f_{2}\left(x_{1}, \cdots, \hat{x}_{i}, \cdots, x_{q+1}\right) \\
& +\frac{1}{q+1} \sum_{i=1}^{q+1}(-1)^{i+1} f_{1}\left(x_{i}\right)\left(d_{x} f_{2}\right)\left(x_{1}, \cdots, \hat{x}_{i}, \cdots, x_{q+1}\right)
\end{aligned}
$$


which proves (19.4) in this case. Assume now that (19.4) holds whenever $f_{1} \in C^{p-1}(L)$. Let now $g_{1} \in C^{1}(L), g_{2} \in C^{p-1}(L)$ and let $f_{1}=g_{1} \square g_{2}$. Then

$$
\begin{aligned}
d_{x}\left(f_{1} \square f_{2}\right) & =d_{x}\left(g_{1} \square g_{2} \square f_{2}\right)=\left(d_{x} g_{1}\right) \square g_{2} \square f_{2}+g_{1} \square d_{x}\left(g_{2} \square f_{2}\right) \\
& =\left(d_{x} g_{1}\right) \square g_{2} \square f_{2}+g_{1} \square\left(d_{x} g_{2}\right) \square f_{2}+g_{1} \square g_{2} \square d_{x} f_{2} \\
& =\left(d_{x} f_{1}\right) \square f_{2}+f_{1} \square\left(d_{x} f_{2}\right) .
\end{aligned}
$$

This implies (19.4) for every $f_{1}$ of the form $g_{1} \square g_{2}$, and by linearity (19.4) holds for all $f_{1}, f_{2}$.

$\operatorname{Ad}(19.5)$. The formula is trivial for $f \in C^{0}(L)$. Assume $f \in C^{1}(L)$. Then (19.5) becomes $-f([[z, x], y])+f([[z, y], x])=-f([z,[x, y]])$ which is a consequence of the Jacobi identity. Assume now that (19.5) holds for $f \in C^{q-1}(L)$. Let $f \in C^{q}(L)$ be of the form $f=f_{1} \square f_{2}$ with $f_{1} \in C^{1}(L)$. Then by (19.4)

$$
\begin{aligned}
d_{[x, y]}\left(f_{1} \square f_{2}\right) & =\left(d_{[x, y]} f_{1}\right) \square f_{2}+f_{1} \square d_{[x, y]} f_{2} \\
& =\left(d_{y} d_{x} f_{1}\right) \square f_{2}-\left(d_{x} d_{y} f_{1}\right) \square f_{2}+f_{1} \square d_{y} d_{x} f_{2}-f_{1} \square d_{x} d_{y} f_{2} \\
& =d_{y} d_{x}\left(f_{1} \square f_{2}\right)-d_{x} d_{y}\left(f_{1} \square f_{2}\right)
\end{aligned}
$$

q.e.d.

Ad(19.6). The formula is trivial for $f \in C^{0}(L)$. Let $f \in C^{1}(L)$ then

$$
-\left(\delta d_{x} f\right)\left(x_{1}, x_{2}\right)=-2^{-1}\left(d_{x} f\right)\left(\left[x_{1}, x_{2}\right]\right)=2^{-1} f\left(\left[\left[x_{1}, x_{2}\right], x\right]\right)
$$

while

$$
\begin{aligned}
-\left(d_{x} \delta f\right)\left(x_{1}, x_{2}\right) & =\delta f\left(\left[x_{1}, x\right], x_{2}\right)+\delta f\left(x_{1},\left[x_{2}, x\right]\right) \\
& =2^{-1} f\left(\left[\left[x_{1}, x\right], x_{2}\right]\right)+2^{-1} f\left(\left[x_{1},\left[x_{2}, x\right]\right]\right)
\end{aligned}
$$

and (19.6) follows from the Jacobi identity. Assume now that (19.6) holds for $f \in C^{q-1}(L)$. Let $f=f_{1} \square f_{2} \in C^{q}(L)$ with $f_{1} \in C^{1}(L)$. Then by (14.2) and (19.4)

$$
\begin{aligned}
\left(d_{x} \delta\right)\left(f_{1} \square f_{2}\right) & =d_{x}\left[\left(\delta f_{1}\right) \square f_{2}-f_{1} \square \delta f_{2}\right] \\
& =\left(d_{x} \delta f_{1}\right) \square f_{2}+\left(\delta f_{1}\right) \square d_{x} f_{2}-\left(d_{x} f_{1}\right) \square \delta f_{2}-f_{1} \square d_{x} \delta f_{2} \\
& =\left(\delta d_{x} f_{1}\right) \square f_{2}+\left(\delta f_{1}\right) \square d_{x} f_{2}-\left(d_{x} f_{1}\right) \square \delta f_{2}-f_{1} \square \delta d_{x} f_{2} \\
& =\delta\left[\left(d_{x} f_{1}\right) \square f_{2}\right]+\delta\left[f_{1} \square d_{x} f_{2}\right]=\delta d_{x}\left(f_{1} \square f_{2}\right)
\end{aligned}
$$

q.e.d.

Ad(19.7). Since $f$ is a cocycle we have

$$
\begin{aligned}
0= & \delta f\left(x, x_{1}, \cdots, x_{q}\right) \\
= & \frac{1}{q+1} \sum_{i=1}^{q}(-1)^{i+1} f\left(\left[x, x_{i}\right], x_{1}, \cdots, \hat{x}_{i}, \cdots, x_{q}\right) \\
& +\frac{1}{q+1} \sum_{i<j}(-1)^{i+j+1} f\left(\left[x_{i}, x_{j}\right], x, x_{1}, \cdots, \hat{x}_{i}, \cdots, \hat{x}_{j}, \cdots, x_{q}\right)
\end{aligned}
$$




$$
\begin{aligned}
= & \frac{1}{q+1}\left(d_{x} f\right)\left(x_{1}, \cdots, x_{q}\right) \\
& -\frac{1}{q+1} \sum_{i<j}(-1)^{i+j+1} f\left(x,\left[x_{i}, x_{j}\right], x_{1}, \cdots, \hat{x}_{i}, \cdots, \hat{x}_{j}, \cdots, x_{q}\right) .
\end{aligned}
$$

If we define the cochain $g \in C^{q-1}(L)$ by

$$
g\left(x_{1}, \cdots, x_{q-1}\right)=q . f\left(x, x_{1}, \cdots, x_{q-1}\right)
$$

the last term above becomes $(\delta g)\left(x_{1}, \cdots, x_{q}\right)$. Therefore $\delta g=d_{x} f$.

With these preliminaries we are ready to prove Theorem 19.1. (19.4) implies

(19.8) If $f_{1}$ and $f_{2}$ are invariant then $f_{1} \square f_{2}$ is invariant.

Proposition (19.5) expresses the fact that $d_{x}$ is a representation of the Lie algebra $L$ with $C^{q}(L)$ as representation space. Proposition (19.6) implies

$$
d_{x} Z^{q}(L) \subset B^{q}(L) \subset Z^{q}(L), \quad d_{x} B^{q}(L) \subset B^{q}(L) .
$$

Since $L$ is semi-simple $Z^{q}(L)$ is the direct sum of $B^{q}(L)$ and some space $T^{q}$ invariant under the representation $x \rightarrow d_{x}$. The proof will be complete if we show that $T^{q}$ consists precisely of all the invariant cocycles. For each $f \in T^{q}$ we have $d_{x} f \in T^{q}$ and $d_{x} f \in E^{q}$. Hence $d_{x} f=0$ and $f$ is invariant. In order to show that all the invariant cocycles are in $T^{a}$ it is sufficient to show that $B^{a}$ contains no invariant cocycles.

Consider now the representation $x \rightarrow d_{x}$ with $C^{q-1}(L)$ as representation space. Since $Z^{q-1}(L)$ is an invariant subspace and $L$ is semi-simple, $C^{q-1}$ is the direct sum of $Z^{q-1}$ and some invariant subspace $D^{q-1}$. Since $\delta: C^{q-1} \rightarrow B^{q}$ maps $C^{q-1}$ onto $B^{q}$ with $Z^{q-1}$ as kernel, it follows that $\delta$ maps $D^{q-1}$ onto $B^{q}$ isomorphically.

Let now $f \in B^{q}(L)$ be an invariant cocycle. Then $f=\delta g$ for some $g \in D^{q-1}$. By (19.6) we have $0=d_{x} f=d_{x} \delta g=\delta d_{x} g$. Hence $d_{x} g \in Z^{q-1}$. But $d_{x} g \in D^{q-1}$, whence $d_{x} g=0$. Consequently $g$ is invariant and by (19.2) $f=\delta g=0$ : This completes the proof.

20. The bilinear form of a representation. Let $P$ be a representation of a Lie algebra $L$ with $V$ as representation space. For $x, y \in L$ define

$$
B(x, y)=\text { Trace }\left(P_{x} P_{y}\right) .
$$

Then $B$ is a bilinear symmetric form on $L$ and

$$
B([x, z], y)=B(x,[z, y])
$$

since

$$
\begin{aligned}
B([x, z], y) & =\text { Trace }\left(P_{[x, z]} P_{y}\right)=\operatorname{Trace}\left(P_{z} P_{x} P_{y}-P_{x} P_{z} P_{y}\right) \\
& =\operatorname{Trace}\left(P_{x}\left(P_{y} P_{z}-P_{z} P_{y}\right)\right)=B(x,[z, y]) .
\end{aligned}
$$


The following lemma will be used in the sequel.

(20.2) If $L$ is semi-simple and the representation $P$ is faithful, the bilinear form associated with $P$ is nonsingular.

This lemma is equivalent to Theorem 1.4 in the paper of Hochschild quoted in footnote 4. This Theorem 1.4 follows easily from Theorem 1.3 of the same paper, which is a well known theorem of Cartan. The matrix which represents the bilinear form of a representation has been considered by Casimir and is sometimes called Casimir's matrix. The fact that it is regular was essential in the algebraic proof given by van der Waerden of the full reducility of representations of semi-simple Lie algebras.

21. The groups $H^{2}(L)$ and $H^{3}(L)$. Using Theorem 19.1 we shall now prove the following theorem.

THEOREM 21.1. If $L$ is a semi-simple Lie algebra over a field of characteristic 0 then $H^{1}(L)=\{0\}, H^{2}(L)=\{0\}$ and $H^{3}(L) \neq\{0\}$.

Proof. The first part of the theorem was proved earlier. In order to prove $H^{2}(L)=0$ it suffices to show that every invariant 2-cocycle $f$ in $L$ is zero. Since $f$ is a cocycle we have

$$
f\left(\left[x_{1}, x_{2}\right], x_{3}\right)-f\left(\left[x_{1}, x_{3}\right], x_{2}\right)+f\left(\left[x_{2}, x_{3}\right], x_{1}\right)=0 .
$$

The first two terms cancel out since $f$ is invariant. Hence $f\left(\left[x_{2}, x_{3}\right], x_{1}\right)=0$. But $[L, L]=L$, whence $f=0$.

In order to prove that $H^{3}(L) \neq 0$ it suffices to exhibit a nonvanishing invariant 3-cochain in $L$. Consider the adjoint representation $\operatorname{ad}(x): y \rightarrow[y, x]$. Define $B(x, y)=$ Trace $(\operatorname{ad}(x) \operatorname{ad}(y))$. Then $B$ is a bilinear symmetric form on $L$. Furthermore by (20.1)

$$
B([x, z], y)=B(x,[z, y]) .
$$

Now define

$$
f\left(x_{1}, x_{2}, x_{3}\right)=B\left(\left[x_{1}, x_{2}\right], x_{3}\right) .
$$

It follows from (21.1) that $f$ is alternating and therefore $f \in C^{8}(L)$. Further

$$
\begin{aligned}
f\left(\left[x_{1}, x\right], x_{2}, x_{3}\right)+f\left(x_{1},\left[x_{2}, x\right], x_{3}\right)+f\left(x_{1}, x_{2},\left[x_{3}, x\right]\right) \\
=B\left(\left[\left[x_{1}, x\right\rfloor, x_{2}\right\rfloor, x_{3}\right)+B\left(\left\lfloor x_{1},\left\lfloor x_{2}, x\right]\right], x_{3}\right)+B\left(\left[x_{1}, x_{2}\right],\left[x_{3}, x\right]\right) \\
=B\left(\left[\left[x_{1}, x_{2}\right], x\right], x_{3}\right)-B\left(\left[x_{1}, x_{2}\right],\left[x, x_{3}\right]\right)=0
\end{aligned}
$$

and $f$ is invariant.

If $f$ is identically zero then $B([x, y], z)$ is identically zero. Since $[L, L]=L$ this implies that $B(x, y)=0$ for all $x, y \in L$. This contradicts (20.2) since the adjoint representation is faithful. 
As a corollary we find:

ThEOREM 21.2. The first and second Betti numbers of a compact semi-simple Lie group are 0 . The third is different from 0.

As a further corollary we prove:

THEOREM 21.3. The n-dimensional sphere $S^{n}$ is a group manifold for the values $n=1,3$ only.

Proof. Suppose that $S^{n}$ is a group manifold and $n>1$. Since $S^{n}$ is compact it can be represented as a Lie group $G$. Since $n>1, S^{n}$ is simply-connected and therefore, by Theorem 16.1, $G$ is semi-simple. The preceding theorem then implies that $n=3$.

22. Relative cohomology groups. Let $L^{\prime}$ be a subalgebra of a Lie algebra $L$ over a field of characteristic 0 . A cochain $f \in C^{q}(L)$ will be called orthogonal to $L^{\prime}$ provided the following two conditions hold

$$
\begin{aligned}
& f\left(x_{1}, \cdots, x_{q}\right)=0 \\
& \text { if } x_{1} \in L^{\prime} \text {, } \\
& d_{x} f=0 \\
& \text { for } x \in L^{\prime} \text {. }
\end{aligned}
$$

These cochains form a subspace $C^{q}\left(L, L^{\prime}\right)$ of $C^{q}(L)$.

(22.3) If $f_{1} \in C^{p}\left(L, L^{\prime}\right)$ and $f_{2} \in C^{q}\left(L, L^{\prime}\right)$ then $f_{1} \square f_{2} \in C^{p+q}\left(L, L^{\prime}\right)$.

Clearly $f_{1} \square f_{2}$ satisfies condition (22.1). Condition (22.2) follows directly from (19.4).

(22.4) If $f \in C^{q}\left(L, L^{\prime}\right)$ then $\delta f \in C^{q+1}\left(L, L^{\prime}\right)$.

Indeed for $x \in L^{\prime}$ we have $(\delta f)\left(x, x_{1}, \cdots, x_{q}\right)=\left(d_{x} f\right)\left(x_{1}, \cdots, x_{q}\right)=0$ if $x \in L^{\prime}$ and $d_{x} \delta f=\delta d_{x} f=0$.

We define $Z^{q}\left(L, L^{\prime}\right)=Z^{q}(L) \cap C^{q}\left(L, L^{\prime}\right)$ and $B^{q}\left(L, L^{\prime}\right)=\delta C^{q-1}\left(L, L^{\prime}\right)$ for $q>0$ and $B^{\circ}\left(L, L^{\prime}\right)=0$. The relative cohomology group of $L \bmod L^{\prime}$ is then defined as the quotient space $H^{q}\left(L, L^{\prime}\right)=Z^{q}\left(L, L^{\prime}\right) / B^{q}\left(L, L^{\prime}\right)$. The cohomology ring $H\left(L, L^{\prime}\right)$ is defined similarly as in $\$ 14$.

Theorem 13.1 combined with Theorem 2.3 and de Rham's theorem then imply the following theorem.

THEOREM 22.1 Let $G$ be a compact connected Lie group, $H$ a closed connected subgroup of $G$ and $M$ the homogenous space $G / H$. Let $L$ be the Lie algebra of $G$ and $L_{H}$ the subalgebra corresponding to $H$. Then the cohomology groups $H^{q}\left(L, L_{H}\right)$ and $H^{a}(M)$ are isomorphic and the rings $H\left(L, L_{H}\right)$ and $H(M)$ are ring isomorphic.

We remark that if $L^{\prime}$ is an ideal in $L$ then condition (22.2) is a consequence of (22.1). Every $q$-cochain of $L$ orthogonal to $L^{\prime}$ may therefore be regarded as a $q$-cochain of the quotient algebra $L / L^{\prime}$ and vice versa. This correspondence commutes with $\delta$ and thus gives $H^{q}\left(L, L^{\prime}\right) \approx H^{q}\left(L / L^{\prime}\right)$. 
Chapter IV. Cohomology groups associated with a Representation

23. Definition of the cohomology groups. Let $L$ be a Lie algebra over a field $K$ of characteristic 0 , and let $P$ be a representation of $L$ by linear transformations of a vector space $V$ of finite dimension over $K$. A $q$-linear alternating mapping of $L$ into $V$ will be called a $q$-dimensional $V$-cochain or shorter $q-V$-cochain. The $q$ - $V$-cochains form a space $C^{q}(L, V)$. By definition $C^{0}(L, V)=V$.

We define a linear mapping $f \rightarrow \delta f$ of $C^{q}(L, V)$ into $C^{q+1}(L, V)$ by the formula

$$
\begin{aligned}
(\delta f) & \left(x_{1}, \cdots, x_{q+1}\right) \\
= & \frac{1}{q+1} \sum_{i=1}^{q+1}(-1)^{i+1} P\left(x_{i}\right) f\left(x_{1}, \cdots, \hat{x}_{i}, \cdots, x_{q+1}\right) \\
& +\frac{1}{q+1} \sum_{i<j}(-1)^{i+j+1} f\left(\left[x_{i}, x_{j}\right], x_{1}, \cdots, \hat{x}_{i}, \cdots, \hat{x}_{j}, \cdots, x_{q+1}\right) .
\end{aligned}
$$

If $q=0$ then $f \in V$ and $\delta f$ is defined by

$$
(\delta f)(x)=P(x) f .
$$

We propose to prove that

$$
\delta \delta f=0 .
$$

A direct proof would be quite cumbersome. We shall give a short proof based on some auxiliary concepts that will be needed in the sequel.

For each $x \in L$ we define a linear mapping $d_{x}: C^{q}(L, V) \rightarrow C^{q}(L, V)$ by setting

$$
\begin{aligned}
\left(d_{x} f\right)\left(x_{1}, \cdots, x_{q}\right)= & P_{x}\left[f\left(x_{1}, \cdots, x_{q}\right)\right]-f\left(\left[x_{1}, x\right], x_{2}, \cdots, x_{q}\right) \\
& -\cdots-f\left(x_{1}, \cdots, x_{q-1}\left[x_{q}, x\right]\right) .
\end{aligned}
$$

If $q=0, d_{x}$ is defined by

$$
d_{x} f=P_{x} f
$$

For $x \in L$ we also define a linear mapping $f \rightarrow f_{x}$ of $C^{q+1}(L, V)$ into $C^{q}(L, V)$ by setting

$$
f_{x}\left(x_{1}, \cdots, x_{q}\right)=(q+1) f\left(x, x_{1}, \cdots, x_{q}\right) .
$$

A direction substitution into the definitions yields the following two formulae, for $x, y \in L$ and $f \in C^{q}(L, V)$

$$
\begin{aligned}
\left(d_{x} f\right)_{y} & =d_{x}\left(f_{y}\right)-f_{[y, x]}, \\
(\delta f)_{x} & =d_{x} f-\delta\left(f_{x}\right) .
\end{aligned}
$$

We shall now prove that 


$$
d_{y} d_{x} f-d_{x} d_{y} f=d_{[x, y]} f
$$

which expresses the fact that $x \rightarrow d_{x}$ is a representation of $L$. If $f \in C^{0}(L, V)$ then (23.7) becomes $P_{y} P_{x} f-P_{x} P_{y} f=P_{[x, y]} f$ which is true since $P$ is a representation. Assume that (23.7) has been proved for all $f \in C^{q-1}(L, V)$ and let $f \in C^{q}(L, V) ; q>0$. Then for every $z \in L$ we have by (23.5)

$$
\begin{aligned}
\left(d_{y} d_{x} f\right)_{z} & =d_{y}\left[\left(d_{x} f\right)_{z}\right]-\left(d_{x} f\right)_{[z, y]} \\
& =d_{y} d_{x}\left(f_{z}\right)-d_{y}\left(f_{[z, x]}\right)-\left(d_{x} f\right)_{[z, y]} \\
& =d_{y} d_{x}\left(f_{z}\right)-\left(d_{y} f\right)_{[z, x]}-f_{\left[\{z, x]_{y}\right]}-\left(d_{x} f\right)_{[z, y]} .
\end{aligned}
$$

Therefore

$$
\begin{aligned}
\left(d_{y} d_{x} f\right)_{z}-\left(d_{x} d_{y} f\right)_{z} & =d_{y} d_{x}\left(f_{z}\right)+f_{[[z, y], x]}-d_{x} d_{y}\left(f_{z}\right)-f_{[[z, x], y]} \\
& =d_{[x, y]}\left(f_{z}\right)-f_{[z,[x, y]]}=\left(d_{[x, y]} f\right)_{z} .
\end{aligned}
$$

Since this holds for each $z \in L,(23.7)$ follows.

Next we prove

$$
\delta d_{x} f=d_{x} \delta f
$$

If $f \in C^{0}(L, V)$ then

$$
\begin{aligned}
\left(d_{x} \delta f\right)\left(x_{1}\right) & =P_{x}\left[\delta f\left(x_{1}\right)\right]-\delta f\left(\left[x_{1}, x\right]\right)=P_{x} P_{x_{1}} f-P_{\left[x_{1}, x\right]} f \\
& =P_{x_{1}} P_{x} f=P_{x_{1}}\left(d_{x} f\right)=\left(\delta d_{x} f\right)\left(x_{1}\right)
\end{aligned}
$$

and (23.8) is proved in this case. Assume now that (23.8) has been proved for all $f \in C^{q-1}(L, V)$ and let $f \in C^{q}(L, V), q>0$. Applying (23.5), (23.6) and (23.7) gives

$$
\begin{aligned}
\left(\delta d_{x} f\right)_{y}-\left(d_{x} \delta f\right)_{y} & =d_{y} d_{x} f-\delta\left[\left(d_{x} f\right)_{y}\right]-d_{x}\left[(\delta f)_{y}\right]+(\delta f)_{[y, x]} \\
& =d_{y} d_{x} f-\delta d_{x} f_{y}-\delta\left(f_{[y, x]}\right)-d_{x}\left[(\delta f)_{y}\right]+d_{[y, x]} f-\delta\left(f_{[y, x]}\right) \\
& =d_{y} d_{x} f-\delta d_{x}\left(f_{y}\right)-d_{x} d_{y} f+d_{x} \delta\left(f_{y}\right)+d_{[y, x]} f \\
& =d_{x} \delta\left(f_{y}\right)-\delta d_{x}\left(f_{y}\right)=0 .
\end{aligned}
$$

Since this holds for every $y \in L$, (23.8) follows.

We can now prove (23.2). If $f \in C^{0}(L, V)$ then $2(\delta \delta f)\left(x_{1}, x_{2}\right)=P_{x_{1}}\left[(\delta f)\left(x_{2}\right)\right]$ $-P_{x_{2}}\left[(\delta f)\left(x_{1}\right)\right]+\delta f\left(\left[x_{1}, x_{2}\right]\right)=P_{x_{1}} P_{x_{2}} f-P_{x_{2}} P_{x_{2}} f+P_{\left[x_{1}, \dot{x}_{2}\right]} f=0$ which proves (23.2) in this case. Assume now that (23.2) holds for all $f \in C^{q-1}(L, V)$ and let $f \in C^{q}(L, V), q>0$. Then by (23.6) and (23.8)

$$
(\delta \delta f)_{x}=d_{x} \delta f-\delta\left[(\delta f)_{x}\right]=d_{x} \delta f-\delta d_{x} f+\delta \delta\left(f_{x}\right)=0 .
$$

Since this holds for every $x \in L$, (23.2) follows.

Having proved that $\delta \delta f=0$ we define the space $Z^{q}(L, P)$ of $q$-P-cocycles as the kernel of the transformation $\delta: C^{q} \rightarrow C^{q+1}$, and the space $B^{q}(L, P)$ of $q-P$-coboundaries as the image $\delta C^{q-1}$. By definition $B^{0}(L, P)=0$. The 
cohomology groups of $L$ over $P$ are then defined as the quotient space $H^{q}(L, P)=Z^{q}(L, P) / B^{q}(L, P)$.

Since $H^{0}(L, P)=Z^{0}(L, P)$ and $(\delta f)(x)=P_{x} f$ for every $x \in L$ and $f \in C^{0}(L, V)$, we conclude that:

(23.9) $H^{0}(L, P)$ is the subspace of the invariant elements of $V$.

For $q>\operatorname{dim} L$ we have $H^{q}(L, P)=0$.

24. The case of semi-simple algebras.

THEOREM 24.1. If $L$ is a semi-simple Lie algebra over a field of characteristic 0 , and $P$ is an irreducible and nontrivial representation of $L$ then $H^{q}(L, P)=0$ for all dimensions $q$.

This theorem shows that in the semi-simple case nothing is gained by studying cohomology groups over representations. In fact any representation $\boldsymbol{P}$ decomposes into irreducible representations and this carries with it a direct decomposition of the cohomology groups. Hence $H^{q}(L, P)$ is isomorphic with the direct sum of several copies of $H^{q}(L)$.

A proof of Theorem 24.1 could be derived from Theorems 2.2 and 10.1 by the application of the unitary trick. The following proof is algebraic (18).

Since the representation $P$ is not trivial, the kernel $L_{0}$ of $P$ is not all of $L$, since $L$ is semi-simple there is an ideal $L_{1}$ in $L$ such that $L$ is the direct sum of $L_{0}$ and $L_{1}$. Let $y_{1}, \cdots, y_{n}$ be a base in $L_{1}$. Since the bilinear form $B(y, z)$ associated with the representation $P$ of $L_{1}$ is nonsingular we can select a dual base $z_{1}, \cdots, z_{n}$ for $L_{1}$ such that

$$
B\left(y_{i}, z_{j}\right)=\delta_{j}^{i} \text {. }
$$

For each $x \in L$ we have $\left[y_{i}, x\right] \in L_{1}$ and therefore $\left[y_{i}, x\right]=\sum_{j=1}^{n} c_{i j} y_{j}$. Similarly $\left[x, z_{i}\right]=\sum_{i=1}^{n} d_{i j} z_{j}$. We shall prove that $c_{i j}=d_{i j}$. Indeed we have $B\left(\left[y_{i}, x\right], z_{j}\right)$ $=B\left(\sum_{k} c_{i k} y_{k}, z_{j}\right)=c_{i j}$ and similarly $B\left(y_{i},\left[x, z_{j}\right]\right)=d_{i j}$. Hence $c_{i j}=d_{i j}$ by formula (20.1). This implies the following two propositions for any $x \in L$ and any linear function $f$ of $L$ to $V$.

$$
\begin{aligned}
& \sum_{i=1}^{n} P_{[y i, x]} f\left(z_{i}\right)=\sum_{i=1}^{n} P_{y i} f\left(\left[x, z_{i}\right]\right), \\
& \sum_{i=1}^{n}\left(P_{y i} P_{\left[x, z_{i}\right]}-P_{[y i, x]} P_{z_{i}}\right)=0 .
\end{aligned}
$$

Consider the linear transformation of $V$ into itself

$$
\Gamma=\sum_{i=1}^{n} P_{y_{i}} P_{z_{i} \cdot}
$$

Since the trace of $\Gamma$ is $n$ it follows that $\Gamma \neq 0$. Further

$\left.{ }^{18}\right)$ Cf. J. H. C. Whitehead, Certain equations in the algebra of a semi-simple infinitesimal group, Quart. J. Math. Oxford Ser. vol. 8 (1937) pp. 220-237. 


$$
\begin{aligned}
\Gamma P_{x} & =\sum_{i=1}^{n} P_{y i} P_{z i} P_{x}=\sum_{i=1}^{n} P_{y i}\left(P_{[x, z i]}-P_{y i} P_{x} P_{z i}\right) \\
& =\sum_{i=1}^{n}\left(P_{y i} P_{[x, z i]}-P_{[y i, x]} P_{z i}+P_{x} P_{y} P_{z i}\right)=P_{x} \Gamma
\end{aligned}
$$

Since $\Gamma$ commutes with each $P_{x}$ the space $\Gamma(V)$ is an invariant subspace of $V$. But the representation $P$ of $L$ is irreducible hence $\Gamma(V)=V$ and $\Gamma$ has an inverse $\Gamma^{-1}$. Clearly $\Gamma^{-1} P_{x}=P_{x} \Gamma^{-1}$.

Let $f \in C^{q}(L, V)$ and let $\Lambda$ be a linear transformation $V \rightarrow V$. Then $\Lambda f \in C^{q}(L, V)$. A direct computation shows that

$$
\begin{aligned}
& (\delta \Lambda f-\Lambda \delta f)\left(x_{1}, \cdots, x_{q+1}\right) \\
& \quad=\frac{1}{q+1} \sum_{i=1}^{q+1}(-1)^{i+1}\left(P_{x j} \Lambda-\Lambda P_{x_{j}}\right) f\left(x_{1}, \cdots, \hat{x}_{i}, \cdots, x_{q+1}\right) .
\end{aligned}
$$

Suppose now that $f$ is a cocycle. Consider the cochains $f_{z_{i}} \in C^{q-1}(L, V)$ and let $g=\sum_{i=1}^{n} P_{y_{i}} f_{x_{i}}$. Then

$$
\delta g=\sum_{i=1}^{n}\left(\delta P_{y i} f_{z i}-P_{y i} \delta f_{z i}\right)+\sum_{i=1}^{n} P_{y i} \delta f_{z i}
$$

and by (23.6)

$$
\delta g=\sum_{i=1}^{n}\left(\delta P_{y i} f_{z i}-P_{y i} \delta f_{z i}\right)+\sum_{i=1}^{n} P_{y i} d z i
$$

Hence by (24.3)

$$
\begin{aligned}
(\delta g)\left(x_{1}, \cdots,\right. & \left.x_{q}\right) \\
= & \sum_{i=1}^{n} \frac{1}{q} \sum_{j=1}^{q}(-1)^{i+1}\left(P_{x j} P_{y i}-P_{y i} P_{x_{j}}\right) f_{z i}\left(x_{1}, \cdots, \hat{x}_{j}, \cdots, x_{q}\right) \\
& +\sum_{i=1}^{n} P_{y i}\left(d_{z i} f\right)\left(x_{1}, \cdots, x_{q}\right) \\
= & \sum_{i=1}^{n} \sum_{i=1}^{q}(-1)^{i+1} P_{\left[y i, x_{j}\right]} f\left(z_{i}, x_{1}, \cdots, \hat{x}_{j}, \cdots, x_{q}\right) \\
& +\sum_{i=1}^{n} P_{y i} P_{z_{i}} f\left(x_{1}, \cdots, x_{q}\right) \\
& -\sum_{i=1}^{n} \sum_{j=1}^{q}(-1)^{j+1} P_{y i} f\left(\left[x_{j}, z_{i}\right], x_{1}, \cdots, \hat{x}_{j}, \cdots, x_{q}\right) .
\end{aligned}
$$

The two double sums cancel in view of (24.1) and therefore $\delta g=\Gamma f$. Since $\Gamma^{-1}$ 
and $P_{x}$ commute, (24.3) implies that $\delta \Gamma^{-1} g=\Gamma^{-1} \delta g=\Gamma^{-1} \Gamma f=f$. Hence $f$ is a coboundary, q.e.d.

25. The group $H^{1}(L, P)$. A detailed discussion of the connections between the first cohomology groups and derivations in a Lie algebra is given by Hochschild( $(4)$.

THEOREM 25.1. A Lie algebra $L$ over a field of characteristic 0 is semi-simple if and only if $H^{1}(L, P)=\{0\}$ for every representation $P$ of $L$.

Proof. It follows from (16.6) and Theorem 24.1 that if $L$ is semi-simple then $H^{1}(L, P)=0$. To prove the converse, consider any representation $P$ of $L$ with representation space $V$ and let $U$ be an invariant subspace of $V$. Choose a subspace $W$ of $B$ (not necessarily invariant) such that $V$ is the direct sum of $U$ and $W$. For each $x \in L$ denote by $\widetilde{P}_{x}$ the transformation $P_{x}$ treated as a linear transformation $W \rightarrow V$. Clearly

$$
\tilde{P}_{x}=Q_{x}+R_{x}
$$

where $Q_{x}: W \rightarrow U$ and $R_{x}: W \rightarrow W$ are linear in both $x \in L$ and $w \in W$. Let $S$ be the space of linear mappings $W \rightarrow U$. Then $Q_{x} \in S$. For $s \in S$ and $x \in L$ define $T_{x} s \in S$ by

$$
T_{x} s=\widetilde{P}_{x} s-s R_{x} .
$$

Since

$$
\tilde{P}_{x_{2}} \tilde{P}_{x_{1}}=\widetilde{P}_{x_{2}} Q_{x_{1}}+\widetilde{P}_{x_{2}} R_{x_{1}}=\widetilde{P}_{x_{2}} Q_{x_{1}}+Q_{x_{2}} R_{x_{1}}+R_{x_{2}} R_{x}
$$

and $\widetilde{P}_{x_{2}} \widetilde{P}_{x_{1}}-\widetilde{P}_{x_{1}} \widetilde{P}_{x_{2}}=\widetilde{P}_{\left[x_{1}, x_{2}\right]}$ it follows that

$$
\begin{aligned}
& R_{\left[x_{1}, x_{2}\right]}=R_{x_{2}} R_{x_{1}}-R_{x_{1}} R_{x_{2}}, \\
& Q_{\left[x_{1}, x_{2}\right]}=T_{x_{2}} Q_{x_{1}}-T_{x_{1}} Q_{x_{2}} .
\end{aligned}
$$

Since

$$
\begin{aligned}
T_{x_{2}} T_{x_{1}} s & =\widetilde{P}_{x_{2}} T_{x_{1}} s-\left(T_{x_{1}} s\right) R_{x} . \\
& =\widetilde{P}_{x_{2}} \tilde{P}_{x} s-\widetilde{P}_{x_{2}} s R_{x_{1}}-\tilde{P}_{x_{1}} s R_{x_{2}}+s R_{x_{2}} R_{x}
\end{aligned}
$$

therefore

$$
\begin{aligned}
T_{x_{2}} T_{x_{1}} s-T_{x_{1}} T_{x_{2}} s & =\tilde{P}_{x_{2}} \tilde{P}_{x_{1}} s-\tilde{P}_{x_{1}} \tilde{P}_{x_{2}} s+s R_{x_{1}} R_{x_{2}}-s R_{x_{2}} R_{x} \\
& =\widetilde{P}_{\left[x_{1}, x_{2}\right]} s-s R_{\left[x_{1}, x_{2}\right]}=T_{\left[x_{1}, x_{2}\right]} s .
\end{aligned}
$$

This shows that $T$ is a representation of $L$ with $S$ as representation space. Then $Q \in C^{1}(L, S)$ and condition (25.1) means that $\delta Q=0$. Since $H^{1}(L, T)=0$, $Q$ is a coboundary. Hence there is a linear transformation $s_{0} \in S$ such that

$$
Q_{x}=T_{x} s_{0}=\widetilde{P}_{x} s_{0}-s_{0} R_{x} .
$$

Hence 


$$
\tilde{P}_{x}=Q_{x}+R_{x}=\tilde{P}_{x} s_{0}-s_{0} R_{x}+R_{x}
$$

which implies

$$
P_{x}\left(w-s_{0}(w)\right)=\left(R_{x}-s_{0} R_{x}\right) w
$$

for each $x \in L, w \in W$.

Define

$$
t(w)=w-s_{0}(w) .
$$

Since $s_{0}(w) \in U, t$ maps $W$ isomorphically onto some subspace $\bar{W}$ of $V$ and $V$ is the direct sum $U+\bar{W}$. Condition (25.2) then becomes $P_{x}(t w)=t\left(R_{x} w\right)$ which shows that $\bar{W}$ is invariant under $P$.

26. Extensions of Lie algebras. In this and the following section it is not assumed that the groundfield is of characteristic 0 . Consequently the factor $1 /(q+1)$ in the formula for the $W$ coboundary will be omitted.

Let $L^{*}$ and $L$ be two Lie algebras and $\phi: L^{*} \rightarrow L$ a homomorphism of $L^{*}$ onto $L$. The pair $\left(L^{*}, \phi\right)$ is called an extension. The kernel $V$ of $\phi$ is called the kernel of the extension. If $[V, V]=0$ the extension is said to have an abelian kernel. An extension is called inessential if there exists a subalgebra $L^{\prime}$ of $L^{*}$ which is mapped by $\phi$ isomorphically onto $L$. As a vector space $L^{*}$ is then the direct sum $L^{\prime}+V$.

The proof of the following theorem is identical with the proof of an analogous theorem established by Hochschild( $\left.{ }^{4}\right)$ for associative algebras.

THEOREM 26.1. If every extension of $L$ with an abelian kernel is inessential, then every extension of $L$ is inessential.

The extensions of $L$ with an abelian kernel will be now studied in greater detail. Let $\left(L^{*}, \phi\right)$ be such an extension with kernel $V$. We select a linear mapping $u: L \rightarrow L^{*}$ such that $\phi u(x)=x$ for each $x \in L$. For each $x \in L$ we then have a mapping $P_{x} v=[v, u(x)]$ of $V$ into itself. Since $[V, V]=0$ it can be easily verified that $P_{x}$ is independent of the choice of $u$. Further $P_{y} P_{x} v-P_{x} P_{y} v$ $=[[v, u(x)], u(y)]-[[v, u(y)], u(x)]=[v,[u(x), u(y)]]=[v, u([x, y])]$ $=P_{[x, y]} v$, and therefore $P$ is a representation of $L$ with $V$ a representation space.

Suppose now that the space $V$ and the representation $P$ of $L$ in $V$ is given. Any pair $\left(L^{*}, \phi\right)$ where $\phi$ is a homomorphism of $L^{*}$ onto $L$ with kernel $V$, such that $[V, V]=0$ and which leads to the given representation $P$, will be called an extension of $L$ by $P$. Two such extensions $\left(L_{1}^{*}, \phi_{1}\right)$ and $\left(L_{2}^{*}, \phi_{2}\right)$ are isomorphic if there is an isomorphism $\theta: L_{1}^{*} \rightarrow L_{2}^{*}$ such that $\theta(v)=v$ for $v \in V$ and that $\phi_{1}=\phi_{2} \theta$.

Given an extension $\left(L^{*}, \phi\right)$ of $L$ by $P$ we select a linear mapping $u: L \rightarrow L^{*}$ with $\phi u(x)=x$. Since $\phi([u(x), u(y)])=[x, y]=\phi u([x, y])$ therefore there is an element $f(x, y) \in V$ such that

$$
[u(x), u(y)]=f(x, y)+u([x, y]) .
$$


The function $f$ is called the factor set corresponding to the function $u$. Clearly $f$ is a $2-V$-cochain.

For $x, y, z \in L$ we have

$$
\begin{aligned}
{[[u(x), u(y)], u(z)] } & =[f(x, y), u(z)]+[u([x, y]), u(z)] \\
& =P_{z} f(x, y)+f([x, y], z)+u([[x, y], z]) .
\end{aligned}
$$

Therefore Jacobi's identity gives

$$
P_{z} f(x, y)+P_{y} f(z, x)+P_{x} f(y, z)+f([x, y], z)+f([z, x], y)+f([y, z], x)=0
$$

or $(\delta f)(x, y, z)=0$. It follows that the factor set $f$ is a 2-P-cocycle.

If $\bar{u}$ is a different linear mapping $\bar{u}: L \rightarrow L^{*}$ such that $\phi \bar{u}(x)=x$, then $h(x)=\bar{u}(x)-u(x) \in V$ and $h$ is a $1-V$-cochain. If $\bar{f}$ is the factor set corresponding to $\bar{u}$ then

$$
\begin{aligned}
\bar{f}(x, y)= & {[\bar{u}(x), \bar{u}(y)]-\bar{u}([x, y])=[u(x), u(y)]+[h(x), u(y)] } \\
& +[u(x), h(y)]+[h(x), h(y)]-u([x, y])-h([x, y]) \\
= & f(x, y)-\left[P_{x} h(y)-P_{y} h(x)+h([x, y])\right]
\end{aligned}
$$

so that

$$
\bar{f}=f-\delta h .
$$

This proves that the cohomology class of $f$ is independent of the choice of $u$. Thus to each extension $\left(L^{*}, \phi\right)$ corresponds a definite element of $H^{2}(L, P)$. Clearly two isomorphic extensions determine the same element of $H^{2}(L, P)$. Given an element of $H^{2}(L, P)$ a corresponding extension can be constructed as follows. Let $f$ be a cocycle belonging to the given element of $H^{2}(L, P)$. As a vector space $L^{*}$ is the direct sum $L+V$, with $\phi(x, v)=x$ and $u(x)=(x, 0)$. Commutation in $L$ is defined by the formula

$$
\left[\left(x_{1}, v_{1}\right),\left(x_{2}, v_{2}\right)\right]=\left(\left[x_{1}, x_{2}\right], P_{x_{2} v_{1}}-P_{x_{2} v_{2}}+f\left(x_{1}, x_{2}\right)\right) \text {. }
$$

It is further easy to see that an extension is inessential if and only if the corresponding cohomology class is zero.

This implies the following theorem.

THEOREM 26.2. The elements of $H^{2}(L, P)$ are in a 1-1 correspondence with isomorphism classes of extensions of $L$ by $P$.

COROLlaRy 26.1. In order that every extension of $L$ over $P$ be inessential it is necessary and sufficient that $H^{2}(L, P)=0$.

Combining this with Theorem 26.1, we find:

ThEOREM 26.3. In order that every extension of a Lie algebra $L$ be inessential it is necessary and sufficient that $H^{2}(L, P)=0$ for every representation $P$.

In view of Theorems 21.1 and 24.1 this condition is satisfied when $L$ is a 
semi-simple Lie algebra over a field of characteristic 0 . Thus we obtain Levi's theorem:

THEOREM 26.4. If $L$ is a semi-simple Lie algebra over a field of characteristic 0 , then every extension of $L$ is inessential.

27. Irreducible extensions. Exterior center. An extension $\left(L^{*}, \phi\right)$ of $L$ by a representation $P$ will be called irreducible if there is no proper subalgebra of $L^{*}$ which is mapped by $\phi$ onto $L$. Let $V_{1}$ be a proper invariant subspace of $V$. This implies that $V_{1}$ is an ideal in $L^{*}$ and $L^{*} / V_{1}$ may also be regarded as an extension of $L$. It is easy to see that $\left(L^{*}, \phi\right)$ is irreducible if and only if the extensions $L^{*} / V_{1}$ are essential for every proper invariant subspace $V_{1}$ of $V$.

We shall now consider extensions $\left(L^{*}, \phi\right)$ corresponding to the trivial representation of $L$ in $V$. Such extensions are called central, because $V$ is then in the center of the extension. These extensions are in a 1-1-correspondence with the element of the cohomology group $H^{2}(L, V)$ (we write $V$ instead of the trivial representation of $L$ in $V$ ).

Any linear map $V \rightarrow V_{1}$ induces in the obvious way a homomorphism $H^{2}(L, V) \rightarrow H^{2}\left(L, V_{1}\right)$. In particular every linear functional $\hat{v}$ on $L$ (with values in the ground field) induces a homomorphism $\hat{v}_{2}$ of $H^{2}(L, V)$ into $H^{2}(L)$. This way every element $e \in H^{2}(L, V)$ determines a homomorphism

$$
\Phi_{e}: \quad \widehat{V} \rightarrow H^{2}(L) .
$$

LEMma 27.1. The correspondence $e \rightarrow \Phi_{\bullet}$ establishes an isomorphism of $H^{2}(L, V)$ with the space of all linear maps $\widehat{V} \rightarrow H^{2}(L)$ of the conjugate space of $V$ into $H^{2}(L)$.

The proof is simple and is left as an exercise to the reader.

Each central extension $E=\left(L^{*}, \phi\right)$ of $L$ with kernel $V$ determines uniquely an element of $H^{2}(L, V)$ and this in turn determines a linear map $\widehat{V} \rightarrow H^{2}(L)$ that we shall denote by $\phi_{E}$. Thus $E \rightarrow \phi_{E}$ establishes a 1-1 correspondence between the equivalence classes of extensions $E$ and the linear maps $\widehat{V} \rightarrow H^{2}(L)$.

THEOREM 27.1. A central extension $E=\left(L^{*}, \phi\right)$ with kernel $V$ is irreducible if and only if the corresponding linear transformation $\phi_{E}: \widehat{V} \rightarrow H^{2}(L)$ is an isomorphism into.

Proof. Suppose that $E$ is not irreducible. There is then a proper subalgebra $L^{\prime}$ of $L^{*}$ which is mapped by $\phi$ onto $L$. Then $V^{\prime}=L^{\prime} \cap V$ is a proper subspace of $V$ and there exists a nonzero element $\hat{v} \in \widehat{V}$ which annihilates $V^{\prime}$. Let $u: L \rightarrow L^{\prime}$ be a linear map such that $\phi u=$ identity. The factor set corresponding to this $u$ will have values in $V^{\prime}$ and therefore will be annihilated by $\tilde{v}$. Hence $\phi_{E} \hat{v}=0$, which shows that $\phi_{E}$ is not an isomorphism.

Conversely, let $\hat{v} \in V, \hat{v} \neq 0$, and $\phi_{E} \bar{v}=0$. Let $V^{\prime}$ be the space of zeros of 
$\hat{v}$ and let $V^{\prime \prime}$ be a subspace of $V$ such that $V$ is the direct sum $V^{\prime}+V^{\prime \prime}$. Let $u: L \rightarrow L^{*}$ be a linear map as before and let $f \in Z^{2}(L, V)$ be the corresponding factor set. The cocycle $f$ determines cocycles $f^{\prime} \in Z^{2}\left(L, V^{\prime}\right)$ and $f^{\prime \prime} \in Z^{2}\left(L, V^{\prime \prime}\right)$ such that $f=f^{\prime}+f^{\prime \prime}$. Since of is a coboundary it follows that $f^{\prime \prime}$ is a coboundary. Let then $f^{\prime \prime}=\delta h$ where $h \in \delta C^{1}\left(L, V^{\prime \prime}\right)$. Replacing the function $u: L \rightarrow L^{*}$ by

$$
\bar{u}(x)=u(x)+h(x)
$$

we find that the factor set is

$$
J=f-\delta h=f^{\prime} .
$$

Then $L^{\prime}=\bar{u}(L)+V^{\prime}$ is a proper subalgebra of $L^{*}$ and $\phi$ maps $L^{\prime}$ onto $L$. Thus $E$ is not irreducible.

Consider now the case when $V=$ conjugate space of $H^{2}(L)$. Let $\phi$ be the natural isomorphism of $\widehat{V}$ with $H^{2}(L)$. Then there is a central extension $E=\left(L^{*}, \phi\right)$ with kernel $V$ such that $\phi_{E}=\phi$. It follows from the previous theorem that $E$ is irreducible. The kernel of $\phi$ is then called the full exterior center of $L$. We can therefore state the following theorem.

THEOREM 27.2. $H^{2}(L)$ is the conjugate space of the full exterior center of the Lie algebra $L$.

28. The relative case. The definition of $\$ 22$ will now be carried over to the case of cohomology groups over a representation. Let $L$ be a Lie algebra over a field of characteristic $0, L^{\prime}$ a subalgebra of $L$ and $P$ a representation of $L$ with the vector space $V$ as representation space.

A $q$ - $V$-cochain $f$ on $L$ will be called orthogonal to $L^{\prime}$ if, as in $\$ 22$,

$$
\begin{array}{rlrl}
f\left(x_{1}, \cdots, x_{q}\right) & =0 & & \text { if } x_{1} \in L^{\prime}, \\
d_{x} f & =0 & \text { for } x \in L^{\prime} .
\end{array}
$$

Condition (28.1) can also be equivalently written as

$$
f_{x}=0
$$

if $x \in L^{\prime}$.

It follows from (23.6) and (23.8) that if $f$ is orthogonal to $L^{\prime}$ then $\delta f$ also is. Consequently if we denote by $C^{q}\left(L, L^{\prime}, V\right)$ the $q$ - $V$-cochains orthogonal to $L^{\prime}$ and define

$$
\begin{aligned}
& Z^{q}\left(L, L^{\prime}, P\right)=Z^{q}(L, P) \cap C^{q}\left(L, L^{\prime}, V\right), \\
& B^{q}\left(L, L^{\prime}, P\right)=\delta C^{q-1}\left(L, L^{\prime}, V\right), \quad B^{0}\left(L, L^{\prime}, P\right)=0, \\
& H^{q}\left(L, L^{\prime}, P\right)=Z^{q}\left(L, L^{\prime}, P\right) / B^{q}\left(L, L^{\prime}, P\right),
\end{aligned}
$$

we arrive at the definition of the cohomology group $H^{q}\left(L, L^{\prime}, P\right)$. As in $\$ 22$ if $L^{\prime}$ is an ideal in $L$ and the representation $P$ is trivial on $L^{\prime}$ then $H^{q}\left(L, L^{\prime}, P\right)$ $\approx H^{a}\left(L / L^{\prime}, P\right)$. 
The definition of $H^{q}\left(L, L^{\prime}, P\right)$ clearly allows for a reformulation of Theorem 13.1.

As a generalization of Theorem 24.1 we prove the following theorem.

THEOREM 28.1. If $L$ is a semi-simple Lie algebra over a field of characteristic $0, L^{\prime}$ is a subalgebra of $L$ and $P$ is an irreducible and nontrivial representation of $L$ then $H^{q}\left(L, L^{\prime}, P\right)=0$ for all dimensions $q$.

Proof. We utilize the notation of the proof of Theorem 24.1. For every $q-P$-cocycle $f$ we constructed a $(q-1)-V$-cochain

$$
g=\sum_{i=1}^{n} P_{y_{i}} f_{z i}
$$

such that

$$
\delta \Gamma^{-1} g=f .
$$

Assume now that $f$ is orthogonal to $L^{\prime}$. We shall then show that $g^{\prime}=\Gamma^{-1} g$ is orthogonal to $L^{\prime}$.

Let $x \in L^{\prime}$. Then

$$
g_{x}=\sum_{i=1}^{n}\left(P_{y_{i}} f_{z i}\right)_{x}=\sum_{i=1}^{n} P_{y_{i}}\left(f_{z_{i}}\right)_{x}=-\sum_{i=1}^{n} P_{y_{i}}\left(f_{x}\right)_{z i}=0 .
$$

Since $\Gamma^{-1}$ is an automorphism of $V$ it follows that

$$
g_{x}^{\prime}=0 \quad \text { for } x \in L^{\prime} .
$$

From (23.6) we deduce

$$
f_{x}=d_{x} g^{\prime}-\delta\left(g_{x}^{\prime}\right) .
$$

Hence for $x \in L^{\prime}, d_{x} g^{\prime}=0$. This combined with (28.3) proves that $g^{\prime}$ is orthogonal to $L^{\prime}$, q.e.d.

Princeton University,

Princeton, N. J.

INDIANA UNIVERSITY,

BLoOMington, IND. 